Research Article

\title{
Stabilization to Exponential Input-to-State Stability of a Class of Neural Networks with Delay by Observer-Based Aperiodic Intermittent Control
}

\author{
Mengyue $\operatorname{Li} \mathbb{D},{ }^{1}$ Biwen $\mathrm{Li}\left(\mathbb{D},{ }^{1}\right.$ and Yuan Wan $\mathbb{D I}^{2,3}$ \\ ${ }^{1}$ College of Mathematics and Statistics, Hubei Normal University, Huangshi 435002, China \\ ${ }^{2}$ Key Laboratory of Urban Land Resources Monitoring and Simulation, MNR, Shenzhen 518034, China \\ ${ }^{3}$ College of Urban and Environmental Science, Hubei Normal University, Huangshi 435002, China
}

Correspondence should be addressed to Biwen Li; lbw20200320@163.com

Received 19 March 2021; Accepted 14 June 2021; Published 6 August 2021

Academic Editor: Shiping Wen

Copyright (c) 2021 Mengyue Li et al. This is an open access article distributed under the Creative Commons Attribution License, which permits unrestricted use, distribution, and reproduction in any medium, provided the original work is properly cited.

This study is devoted to investigating the stabilization to exponential input-to-state stability (ISS) of a class of neural networks with time delay and external disturbances under the observer-based aperiodic intermittent control (APIC). Compared with the general neural networks, the state of the neural network investigated is not yet fully available. Correspondingly, an observer-based APIC is constructed, and moreover, neither the observer nor the controller requires the information of time delay. Then, the stabilization to exponential ISS of the neural network is realized severally by the observer-based time-triggered APIC (T-APIC) and the observer-based event-triggered APIC (E-APIC), and corresponding criteria are given. Furthermore, the minimum activation time rate (MATR) of the observer-based T-APIC and the observer-based E-APIC is estimated, respectively. Finally, a numerical example is given, which not only verifies the effectiveness of our results but also shows that the observer-based E-APIC is superior to the observer-based T-APIC and the observer-based periodic intermittent control (PIC) in control times and the minimum activation time rate, and the function of the observer-based T-APIC is also better than the observer-based PIC.

\section{Introduction}

Neural network, a mathematical model for information processing, can better simulate the working mechanism of the brain and plays a crucial role in artificial intelligence. The research on the dynamic behaviors of the neural network is the premise of the successful application of the neural network in many fields, such as associative memory, optimization problems, image recognition, different learning tasks, and so on [1-7]. In recent years, the research on the dynamic behaviors of the neural network mainly includes synchronization, stability, periodicity, and other dynamic behaviors, and some new and excellent results are emerging continuously $[2,8-11]$.

As an important dynamic behavior of the neural network, stability has always been concerned by scholars. At present, the research on stability mainly includes asymptotic stability, exponential stability, Lagrange stability, and other stabilities [12-18]. Exponential stability has attracted much attention due to its advantages of fast convergence speed and fast achieving the expected goal in practical application. By using a new multiple Lyapunov function and linear matrix inequalities, Lian and Zhang [14] discussed the exponential stability of a class of uncertain switched Cohen-Grossberg neural networks with interval time-varying, distributed delay, and average dwell time. In [16], a class of hybrid neural networks with inertia term and mixed time-varying delays has been noticed. Then, by establishing appropriate Lyapunov functionals and inequality technique, some sufficient conditions have been given to implement exponential stability of the system.

For one thing, as a result of the limitations of existing technical means, the switching speed of hardware devices is limited, which leads to the time delay phenomenon 
ubiquitous and inevitable in all kinds of engineering application. The existence of time delay is often one of the important factors affecting the stability of dynamic systems. For another, the system will inevitably be affected by external disturbances in practical application. For example, the communication signals will be interfered by other signals in the process of transmission, the machine will be affected by vibration in the process of operation, etc. Therefore, it is more practical to study the stability of neural networks with time delay and external disturbances. Halanay inequality and the generalization of Halanay-type inequality [8] are commonly used in the study of time delay systems. Input-tostate stability has attracted much attention because it can describe the impact of external disturbances on the dynamic system. In other words, input-to-state stability means that the system is probably asymptotically stable without disturbances, and when the input of the system is bounded, the response of the system is also bounded. In [19], the input-tostate stability, a class of delayed dynamical systems with bounded disturbances, was concerned. The input-to-state stability analysis of a class of impulsive stochastic CohenGrossberg neural networks with mixed delays was discussed in [20]. Based on stochastic analysis theory, impulse theory, Itô formula, Dynkin formula, and so on, the mean square exponential input-to-state stability of systems with/without impulsive control was obtained.

In order to ensure the stable operation of the system, it is frequently necessary to exert external control on the system. It is a remarkable fact that the control in some practical applications is frequently discontinuous, such as vehicle control, the management of wind power generation, refrigeration system of a refrigerator, and the orbit adjustment of spacecraft. In some practical applications, as mentioned above, discontinuous control is not only less costly but also more practical than continuous control. Intermittent control and impulsive control belong to discontinuous control. Intermittent control and impulsive control are similar but different. Intermittent control is executed in some time intervals, while impulsive control is activated at instants. At present, numerous researchers have studied the impulsive control based on the stability of the impulsive system and achieved good results [21-25]. Yet, in some practical applications, it may be difficult to complete a control task in a flash, for example, vehicle acceleration and deceleration control, where the control is intermittent rather than instantaneous. If not, the movement of the vehicle will bump and shock. In this situation, intermittent control is obviously better than impulsive control. As far as we know, compared with impulsive control, the research results of intermittent control are relatively few.

In this study, we will consider studying the ISS of the neural network by intermittent control. In recent years, periodic intermittent control has been gradually used to realize some common dynamic behaviors of the neural network [26]. However, in some practical applications, demand of periodicity is unnecessary and unreasonable, such as the wind power generation mentioned above. The aperiodic intermittent control has the characteristics that the duration of each control and the time interval of two adjacent controls are not fixed, so its flexibility is better and practicability is stronger. Apparently, in order to eliminate the limitation of periodicity, aperiodic intermittent control is a better choice. What is noteworthy is that multitudinous scholars focus on the synchronization problem of the system under aperiodic intermittent control [27-29], while the research on the stability is relatively less. Using the condition of converse average dwell time, Liu et al. [30] designed a time-triggered aperiodic intermittent controller to realize exponential input-to-state stability of the continuous-time system. In addition, to reduce the conservatism existing in the time-triggered aperiodic intermittent controller, Liu et al. proposed the event-triggered aperiodic intermittent control. It is worth noting that the state of the system discussed in [30] is measurable, and the controllers use the real state of the system to control. However, due to some physical limitations or measurement technology limitations, the state of the system is often not completely obtained, which will lead to the failure of the feedback control based on the state of the system. Inspired by [30], it is very interesting to study the exponential ISS of the neural network by using the aperiodic intermittent control when the system state is not measurable. Building a state observer is one of the common methods to solve the problem of unmeasurable system state, and many excellent and interesting results have been obtained in many fields [31-33]. Furthermore, as far as we know, almost no scholars use aperiodic intermittent control to achieve the exponential ISS of neural networks with time delay and external disturbances when the system state is unmeasurable.

According to the above elaboration, this study mainly focuses on exponential input-to-state stability of a class of neural networks with time delay and external disturbances under observer-based aperiodic intermittent control. The main work of this study is summarized as follows:

(1) A state observer independent of time delay information is constructed, which is more practical because time delay information is often not and is not easy to obtain completely in some practical applications

(2) Combining the time-triggered mechanism with the aperiodic intermittent control based on the state observer, an observer-based time-triggered controller is put forward. Then, the activation time and control duration of the controller are designed by using the infimum of the single activation time rate of the controller and the converse average dwell time method to guarantee the exponential ISS of the neural network. Furthermore, the minimum activation time rate of the controller is estimated.

(3) In order to reduce the conservatism existing in the observer-based time-triggered aperiodic intermittent control, an observer-based event-triggered control mechanism is proposed. Meanwhile, the criterion to 
ensure the exponential input-to-state stability of the studied network is given, and the minimum activation time rate of the controller is evaluated.

The arrangement of the rest of this study is as follows. The second part is the symbol description and the construction of neural networks. The third and fourth parts are the derivation of the results obtained in this study. In the fifth part, a numerical example is given to verify the effectiveness of the results. The conclusion and prospect are given in the sixth part.

\section{Preliminaries}

Let $\mathbb{R}$ and $\mathbb{R}^{n}$ denote the set of real numbers and the $n$-dimensional real vector space, respectively. $I$ represents the identity matrix, $\mathbb{R}_{+}=[0, \infty)$, and $\mathbb{N}=\{0,1, \ldots, n\}$. $\lambda_{\max }(Q)$ stands for the maximum eigenvalue of the symmetric matrix Q. For a matrix $A=\left(a_{i j}\right)_{n \times m}$, its norm is defined as $\|A\|=\left[\lambda_{\max }\left(A^{T} A\right)\right]^{(1 / 2)}$. Denoted by $C($ $\left.[-\tau, 0], \mathbb{R}^{n}\right)$, the Banach space composed of all continuous vector-valued functions $\varphi:[-\tau, 0] \longrightarrow \mathbb{R}^{n}$.

The symbol $L_{\infty, \text { loc }}$ represents the set of Lebesgue measurable functions, which are composed of locally essentially bounded functions $\iota: \mathbb{R}_{+} \longrightarrow \mathbb{R}^{n}$. For $\iota \in L_{\infty, \text { loc }}$, set $\|\iota\|_{\left[s_{1}, s_{2}\right]}=\operatorname{ess} \sup _{s_{1} \leq t \leq s_{2}}\{\|\iota(t)\|\} \quad$ and $\quad\|\iota\|_{\infty}=\operatorname{ess} \sup _{t \in \mathbb{R}_{+}}$ $\{\|\iota(t)\|\}$.

In this study, we discuss the following neural network model with time delay and external disturbances.

$$
\left\{\begin{array}{l}
\dot{x}(t)=-C x(t)+A f(x(t))+B g(x(t-\tau))+u_{1}(t)+\omega(t), t \geq t_{0}, \\
x\left(t_{0}+\theta\right)=\varphi(\theta) \in C\left([-\tau, 0], \mathbb{R}^{n}\right),
\end{array}\right.
$$

where $x(t)=\left(x_{1}(t), x_{2}(t), \ldots, x_{n}(t)\right)^{T} \in \mathbb{R}^{n}$ is the state vector of the neural network; $x\left(t_{0}+\theta\right)=\varphi(\theta), \theta \in[-\tau, 0]$ is the initial value condition of $(1) ; f(x(t))=\left(f_{1}\left(x_{1}(t)\right)\right.$, $\left.f_{2}\left(x_{2}(t)\right), \ldots, f_{n}\left(x_{n}(t)\right)\right)^{T}$ and $g(x(t-\tau))=\left(g_{1}\left(x_{1}(t-\right.\right.$ $\left.\tau)), g_{2}\left(x_{2}(t-\tau)\right), \ldots, g_{n}\left(x_{n}(t-\tau)\right)\right)^{T}$ are the activation functions at time $t$ and delay time $t-\tau ; C=\operatorname{diag}\left\{c_{1}\right.$, $\left.c_{2}, \ldots, c_{n}\right\}$ is the real diagonal matrix, where $c_{i}>0, i=1,2$, $\ldots, n ; A, B$ are the weight matrices with corresponding dimensions; the control input of the neural network is represented by $u_{1}(t) ; \omega(t) \in L_{\infty \text {,loc }}$ is the external disturbances; $\tau>0$ is a transmission delay.

Suppose that (1) with $u=0$ is not ISS. The purpose of this study is to design the APIC to ensure that (1) is exponential ISS. Furthermore, considering that, in a great deal of engineering applications, due to some physical limitations or other reasons, the system state may be extremely difficult or almost impossible to obtain completely, which leads to the malfunction of feedback control based on the real state of the system. Therefore, we construct a state observer (2):

$$
\left\{\begin{array}{l}
\dot{\tilde{x}}(t)=-C \tilde{x}(t)+A f(\widetilde{x}(t))+B g(\widetilde{x}(t))+u_{2}(t), \quad t \geq t_{0}, \\
\tilde{x}\left(t_{0}\right)=\psi \in \mathbb{R}^{n},
\end{array}\right.
$$

where $\tilde{x}(t) \in \mathbb{R}^{n}$ is the estimation of state $x(t)$; $\tilde{x}\left(t_{0}\right)=\psi \in \mathbb{R}^{n}$ is the initial value condition of (2).

Remark 1. Because the state value is unknown, it is very likely that the time delay cannot be known, so it is uncertain whether to adopt time-varying delay, distributed constant delay, or other time-delay in the observer system. Thus, the time delay information is not used to design an observer to estimate the state of (1). According to this, we consider the above state observer. Moreover, when the time delay is measurable, it can be similar to the following discussion about the unmeasurable time delay. Therefore, the study only considers the case where the delay information is unmeasurable.

Let $t_{i}$ denote the starting instant of the controller and $\vartheta_{i}$ represent the control duration of the controller. Then, $u_{2}(t)$ and $u_{1}(t)$ are designed as

$$
\begin{aligned}
& u_{2}(t)= \begin{cases}K_{2} \tilde{x}(t), & t_{i} \leq t<t_{i}+\vartheta_{i}, \\
0, & t_{i}+\vartheta_{i} \leq t<t_{i+1},\end{cases} \\
& u_{1}(t)= \begin{cases}K_{1} \tilde{x}(t), & t_{i} \leq t<t_{i}+\vartheta_{i}, \\
0, & t_{i}+\vartheta_{i} \leq t<t_{i+1},\end{cases}
\end{aligned}
$$

where $K_{2}$ and $K_{1}$ are the control gain matrices.

Remark 2. Considering the immeasurability of system state, the real state of the system cannot be used as feedback control; hence, we consider to regulate and control observer (2) and real system (1) by the observation value. Furthermore, the control input $u_{1}(t)$ of $(1)$ is APIC. Hence, in order to construct a proper error system, the control input $u_{2}(t)$ of observer (2) is also designed to be APIC.

Remark 3. Compared with the periodic intermittent control (PIC) proposed in [26], where

$$
u(t)= \begin{cases}K x(t), & i T \leq t<i T+\vartheta \\ 0, & i T+\vartheta \leq t<(i+1) T,\end{cases}
$$

the time span of each control in APIC is more flexible. In the above formula, $T>0$ stands for the period of control and $0<\vartheta<T$ denotes activation time of $u(t)$.

Combining (2) and (3), observer (2) can be rewritten as

$$
\left\{\begin{array}{l}
\dot{\tilde{x}}(t)=-\left(C-K_{2}\right) \tilde{x}(t)+A f(\tilde{x}(t))+B g(\tilde{x}(t)), \quad t_{i} \leq t<t_{i}+\vartheta_{i}, \\
\dot{\tilde{x}}(t)=-C \tilde{x}(t)+A f(\tilde{x}(t))+B g(\tilde{x}(t)), \quad t_{i}+\vartheta_{i} \leq t<t_{i+1}, i \in \mathbb{N}, \\
\tilde{x}\left(t_{0}\right)=\psi \in \mathbb{R}^{n} .
\end{array}\right.
$$

Similarly, combining (1) and (4), system (1) becomes the following form: 


$$
\begin{cases}\dot{x}(t)=-C x(t)+K_{1} \tilde{x}(t)+A f(x(t))+B g(x(t-\tau))+\omega(t), & t_{i} \leq t<t_{i}+\vartheta_{i}, \\ \dot{x}(t)=-C x(t)+A f(x(t))+B g(x(t-\tau))+\omega(t), & t_{i}+\vartheta_{i} \leq t<t_{i+1}, i \in \mathbb{N}, \\ x\left(t_{0}+\theta\right)=\varphi(\theta) \in C\left([-\tau, 0], \mathbb{R}^{n}\right) . & \end{cases}
$$

Let $e(t)=x(t)-\tilde{x}(t)$ denote the error between neural network (1) and observer (2); then, the dynamic of the error system can be described as

$$
\begin{cases}\dot{e}(t)=-C e(t)+A[f(x(t))-f(\tilde{x}(t))]+\left(K_{1}-K_{2}\right) \tilde{x}(t)+B[g(x(t-\tau))-g(\tilde{x}(t))]+\omega(t), & t_{i} \leq t<t_{i}+\vartheta_{i}, \\ \dot{e}(t)=-C e(t)+A[f(x(t))-f(\tilde{x}(t))]+B[g(x(t-\tau))-g(\tilde{x}(t))]+\omega(t), & t_{i}+\vartheta_{i} \leq t<t_{i+1} \\ e\left(t_{0}+\theta\right)=\tilde{\phi}(\theta)=\varphi(\theta)-\psi \in C\left([-\tau, 0], \mathbb{R}^{n}\right), & \end{cases}
$$

where $i \in \mathbb{N}$.

It is noteworthy that the real system (1) can be stable when both the observation system (2) and error system (8) are stable. Hence, we consider realizing the stability of (1) by realizing the stability of the augmented system composed of (2) and (8).

Let $z(t)=\left(\tilde{x}^{T}(t), e^{T}(t)\right)^{T} \quad$ and $\quad z\left(t_{0}+\theta\right)=$ $\Phi(\theta)=\left(\psi^{T}, \widetilde{\phi}^{T}(\theta)\right)^{T} \in C\left([-\tau, 0], \mathbb{R}^{2 n}\right)$. Then, combining (6) and (8), the following argument system can be obtained:

$$
\begin{cases}\dot{z}(t)=C_{1} z(t)+A_{1} F(t)+B_{1} G(t-\tau)+W(t), & t_{i} \leq t<t_{i}+\vartheta_{i}, \\ \dot{z}(t)=C_{2} z(t)+A_{1} F(t)+B_{1} G(t-\tau)+W(t), & t_{i}+\vartheta_{i} \leq t<t_{i+1}, i \in \mathbb{N}, \\ z\left(t_{0}+\theta\right)=\Phi(\theta) \in C\left([-\tau, 0], \mathbb{R}^{2 n}\right), & \end{cases}
$$

where

$$
\begin{aligned}
W(t) & =\left(0, \omega^{T}(t)\right)^{T}, \\
F(t) & =\left(f^{T}(\tilde{x}(t)), f^{T}(x(t))-f^{T}(\tilde{x}(t))\right)^{T}, \\
G(t) & =\left(g^{T}(\tilde{x}(t)), g^{T}(x(t-\tau))-g^{T}(\tilde{x}(t))\right)^{T}, \\
A_{1} & =\left[\begin{array}{ll}
A & 0 \\
0 & A
\end{array}\right], \\
B_{1} & =\left[\begin{array}{ll}
B & 0 \\
0 & B
\end{array}\right], \\
C_{1} & =\left[\begin{array}{cc}
-C+K_{2} & 0 \\
K_{1}-K_{2} & -C
\end{array}\right], \\
C_{2} & =\left[\begin{array}{cc}
-C & 0 \\
0 & -C
\end{array}\right] .
\end{aligned}
$$

Assume that $z(t)=z\left(t, t_{0}, \Phi\right)$ is the solution of (9) with the initial condition $z\left(t_{0}+\theta\right)=\Phi(\theta) \in C\left([-\tau, 0], \mathbb{R}^{2 n}\right)$.

Definition 1. [30]. System (1) is exponential input-to-state stable via observer-based APIC (4) if system (9) is exponentially ISS with respect to $\omega(t)$, i.e., there exist $M>0, r>0$, and function $\Psi \in \mathscr{K}_{\infty}$, such that the solution $z(t)$ of (9) satisfies

$$
\|z(t)\| \leq M\left\|z\left(t_{0}\right)\right\| e^{-r\left(t-t_{0}\right)}+\Psi\left(\|\omega\|_{\left[t_{0}, t\right]}\right), \quad t \geq t_{0}
$$

Now, we give the following assumptions and lemmas which are extremely necessary for our follow-up derivation.

Assumption 1. Functions $f(\cdot), g(\cdot) \in \mathbb{R}^{n}$ with $f(0)=0$, and $g(0)=0$ are Lipschitz continuous, that is, $\forall x_{i}, y_{i} \in \mathbb{R}$; there exist $l_{1 i}>0, \widetilde{l}_{2 i}>0$ satisfy

$$
\begin{aligned}
\left|f_{i}\left(x_{i}\right)-f_{i}\left(y_{i}\right)\right| \leq l_{1 i}\left|x_{i}-y_{i}\right|, & i=1,2, \ldots, n, \\
\left|g_{i}\left(x_{i}\right)-g_{i}\left(y_{i}\right)\right| \leq \widetilde{l}_{2 i}\left|x_{i}-y_{i}\right|, & i=1,2, \ldots, n .
\end{aligned}
$$

According to Assumption 1 , let $l_{1}=\max \left\{l_{11}, l_{12}, \ldots, l_{1 n}\right\}$ and $l_{2}=\max \left\{\widetilde{l}_{21}, \widetilde{l}_{22}, \ldots, \widetilde{l}_{2 n}\right\}$.

Assumption 2. Suppose there are matrices $K_{1}, K_{2}$ and positive constants $g_{1}, g_{2}$ that satisfy the following conditions:

$$
\begin{array}{r}
C_{1}+C_{1}^{T}+A_{1} A_{1}^{T}+B_{1} B_{1}^{T}+\left(l_{1}^{2}+3 l_{2}^{2}+g_{1}\right) I \leq 0, \\
2 C_{2}+A_{1} A_{1}^{T}+B_{1} B_{1}^{T}+\left(l_{1}^{2}+3 l_{2}^{2}-g_{2}\right) I \leq 0 .
\end{array}
$$

Lemma 1 (see [34]). For any $\mathscr{X}, \mathscr{Y} \in \mathbb{R}^{n}$, we have

$$
\mathscr{X}^{T} \mathscr{Y}+\mathscr{Y}^{T} \mathscr{X} \leq \varepsilon \mathscr{X}^{T} \mathscr{X}+\varepsilon^{-1} \mathscr{Y}^{T} \mathscr{Y},
$$

where $\varepsilon$ is any positive constant. 
Lemma 2. Let $V:\left[t_{0}-\tau,+\infty\right) \longrightarrow[0,+\infty)$ and $E:\left[t_{0},+\infty\right) \longrightarrow[0,+\infty)$. If the derivative of $V$ with respect to $t$ satisfies the following inequality:

$$
\dot{V}(t) \leq \alpha V(t)+\beta V(t-\tau)+E(t), \quad t \geq t_{0},
$$

where $\alpha \in \mathbb{R}$ and $\beta>0, \tau>0$, then, for any $t \geq t_{0}$, the following result can be obtained:

$$
V(t) \leq\left(V\left(t_{0}\right)+\int_{t_{0}}^{t} e^{-r\left(s-t_{0}\right)} E(s) \mathrm{d} s\right) e^{r\left(t-t_{0}\right)},
$$

when $-r+\alpha+\beta \max \left\{e^{-r \tau}, 1\right\} \leq 0$.

Proof. Let

$$
\bar{V}(t)= \begin{cases}V(t), & t \in\left[t_{0}-\tau, t_{0}\right), \\ V(t) e^{-r\left(t-t_{0}\right)}, & t \geq t_{0} .\end{cases}
$$

From (16) and (18), it follows that

$$
\dot{\bar{V}}(t)=\dot{V}(t) e^{-r\left(t-t_{0}\right)}-r V(t) e^{-r\left(t-t_{0}\right)} \leq(\alpha-r) V(t) e^{-r\left(t-t_{0}\right)}+\beta V(t-\tau) e^{-r\left(t-t_{0}\right)}+e^{-r\left(t-t_{0}\right)} E(t), \quad t \geq t_{0}
$$

(19) is discussed as follows:

(a) If $t-\tau \in\left[t_{0}-\tau, t_{0}\right)$, then $V(t-\tau)=\bar{V}(t-\tau)$, $t-t_{0} \in[0, \tau)$, and we have

$$
\dot{\bar{V}}(t) \leq(\alpha-r) \bar{V}(t)+\beta \bar{V}(t-\tau) \max \left\{e^{-r \tau}, 1\right\}+e^{-r\left(t-t_{0}\right)} E(t), \quad t \geq t_{0} .
$$

(b) If $t-\tau \in\left[t_{0},+\infty\right)$, then

$$
\dot{\bar{V}}(t) \leq(\alpha-r) \bar{V}(t)+\beta V(t-\tau) e^{-r\left(t-\tau-t_{0}\right)} e^{-r \tau}+e^{-r_{1}\left(t-t_{0}\right)} E(t) \leq(\alpha-r) \bar{V}(t)+\beta \bar{V}(t-\tau) \max \left\{e^{-r \tau}, 1\right\}+e^{-r\left(t-t_{0}\right)} E(t), \quad t \geq t_{0} .
$$

Hence, for any $t \geq t_{0}$, we get

$\dot{\bar{V}}(t) \leq(\alpha-r) \bar{V}(t)+\beta \max \left\{e^{-r \tau}, 1\right\} \bar{V}(t-\tau)+e^{-r\left(t-t_{0}\right)} E(t)$.

Let

$$
U(t)= \begin{cases}\bar{V}(t), & t \in\left[t_{0}-\tau, t_{0}\right), \\ \bar{V}(t)-\int_{t_{0}}^{t} e^{-r\left(s-t_{0}\right)} E(s) \mathrm{d} s, & t \geq t_{0},\end{cases}
$$

then, for any $t \geq t_{0}$,

$$
\begin{aligned}
\dot{U}(t) & \leq(\alpha-r) \bar{V}(t)+\beta \max \left\{e^{-r \tau}, 1\right\} \bar{V}(t-\tau) \\
& \leq\left(\alpha-r+\beta \max \left\{e^{-r \tau}, 1\right\}\right) \sup \{\bar{V}(t), \bar{V}(t-\tau)\} \\
& \leq 0,
\end{aligned}
$$

since $-r+\alpha+\beta \max \left\{e^{-r \tau}, 1\right\} \leq 0$. Therefore, it follows that

$$
U(t) \leq U\left(t_{0}\right)=\bar{V}\left(t_{0}\right)
$$

for any $t \geq t_{0}$. Then, combining (23), for any $t \geq t_{0}$, we have

$$
\bar{V}(t)-\int_{t_{0}}^{t} e^{-r\left(s-t_{0}\right)} E(s) \mathrm{d} s \leq \bar{V}\left(t_{0}\right)=V\left(t_{0}\right),
$$

and so

$$
\bar{V}(t) \leq V\left(t_{0}\right)+\int_{t_{0}}^{t} e^{-r\left(s-t_{0}\right)} E(s) \mathrm{d} s,
$$

which means (17) holds. The deducing procedure is finished.

Remark 4. Lemma 2 obtained the variation of $V(t)$ in the interval $\left[t_{0},+\infty\right)$ on the premise that the derivative of $V(t)$ satisfies condition (16). For the variation of $V(t)$ in the interval $\left[t_{0}-\tau, t_{0}\right), \quad$ it is clear that $V(t) \leq \sup _{\zeta \in[-\tau, 0]} V\left(t_{0}+\zeta\right)$. Moreover, the value of $V(t)$ in the interval $\left[t_{0}-\tau, t_{0}\right)$ can be regarded as the initial value of differential equation (16). Because the initial value is arbitrary and is predetermined, it is not necessary to know the variation of $V(t)$ exactly when $t \in\left[t_{0}-\tau, t_{0}\right)$. Instead, we should pay attention to the variation of $V(t)$ when $t \geq t_{0}$, and the function $V(t)$ satisfies a certain differential equation. 
For the stability of system (9) under the observer-based APIC $\left(K_{1}, K_{2},\left\{t_{i}\right\},\left\{\vartheta_{i}\right\}\right)$, let $K_{1}$ and $K_{2}$ satisfy (13) and (14) first. The next step is how to construct $\left\{t_{i}\right\}$ and $\left\{\vartheta_{i}\right\}$.

To begin with, ensure that the observer-based APIC $\left(K_{1}, K_{2},\left\{t_{i}\right\},\left\{\vartheta_{i}\right\}\right)$ is designed with the following characteristics:

(1) No Zeno behavior

(2) The controller works intermittently

$\left\{t_{i}\right\}$ should satify the following condition:

$$
\begin{array}{r}
t_{i}<t_{i+1}, \quad \forall i \in \mathbb{N} ; \\
\lim _{i \longrightarrow \infty} t_{i}=\infty .
\end{array}
$$

The duration of control $\vartheta_{i}$ should satify

$$
0<\vartheta_{i}<\Delta_{i} \triangleq t_{i+1}-t_{i}, \quad \forall i \in \mathbb{N},
$$

and let $\vartheta_{\text {min }}=\min _{i \in \mathbb{N}}\left\{\vartheta_{i}\right\}>0, \vartheta_{\max }=\max _{i \in \mathbb{N}}\left\{\vartheta_{i}\right\}<\infty$.

Therefore, the problem of stabilization to ISS by observer-based APIC formulated the issue of minimum activation time rate (MATR):

$$
R_{\min }=\min _{\left\{t_{i}\right\},\left\{\vartheta_{i}\right\}}\left\{\lim _{i \rightarrow \infty} \inf \frac{\sum_{j=0}^{i} \vartheta_{j}}{\sum_{j=0}^{i} \Delta_{j}}\right\},
$$

where $t_{i}$ satisfies (28) and $\vartheta_{i}$ satisfies (29). Additionally, for a controller, if the duration of each control is $\vartheta_{i}$, then the activation time rate of the controller during $[t, s)$ is defined as $R(t)=\left(\sum_{i=0}^{N-1} \vartheta_{i} /(s-t)\right) \times 100 \%$, where the notation $N$ signifies the number of times the controller is activated during the period.

\section{Stabilization to Exponential ISS via Observer- Based T-APIC}

In this section, we devote ourselves to achieving exponential ISS of system (1) via the observer-based T-APIC and consider the following two cases to design the observerbased T-APIC.

Case 1. $\left\{t_{i}\right\}$ is given to satisfy (28). Here, we design the observer-based time-triggered aperiodic intermittent control by using infimum of single activation time rate $\gamma_{\text {inf }} \triangleq \inf _{i \in \mathbb{N}}\left\{\vartheta_{i} / \Delta_{i}\right\}$ and the converse average dwell time, respectively, and give the criterion of exponential input-tostate stability of neural network (1) under the control. In addition, we estimate the minimum activation time rate $R_{\min }$ of the control.

Theorem 1. Suppose that $\left\{t_{i}\right\}$ is given to satisfy (28) and Assumptions 1 and 2 hold. Moreover, there exist $\widetilde{r}_{1}<0$ and $r_{2}>0$, such that

$$
\begin{array}{r}
-\widetilde{r}_{1}-\left(g_{1}-\varepsilon_{1}\right)+4 l_{2}^{2} e^{-\tilde{r}_{1} \tau} \leq 0, \\
-r_{2}+\left(g_{2}+\varepsilon_{2}\right)+4 l_{2}^{2} \leq 0,
\end{array}
$$

where $\varepsilon_{1}$ and $\varepsilon_{2}$ are the appropriate constants satisfying $0<\varepsilon_{1}<g_{1}$ and $0<\varepsilon_{2}<g_{2}$. Then, system (1) can achieve stabilization to exponential ISS under observer-based T-APIC (4), if $\left\{\vartheta_{i}\right\}$ satisfies (29) and the following condition:

$$
R_{u}^{*}=\frac{r_{2}}{r_{1}+r_{2}}<\gamma_{\text {inf }}=\inf _{i \in \mathbb{N}}\left\{\frac{\vartheta_{i}}{\Delta_{i}}\right\}<1,
$$

where $r_{1}=\left|\widetilde{r}_{1}\right|$. In addition, we have

$$
R_{\min }>R_{u}^{*} \text {. }
$$

Proof. Let $V(z(t))=z^{T}(t) z(t)$.

For any $t_{i} \leq t<t_{i}+\vartheta_{i}$, computing the derivative of $V(z(t))$ with respect to the time $t$, we get that

$$
\begin{aligned}
\dot{V}(z(t)) & =2 z^{T}(t) \dot{z}(t) \\
& =2 z^{T}(t) C_{1} z(t)+2 z^{T}(t) A_{1} F(t)+2 z^{T}(t) B_{1} G(t-\tau)+2 z^{T}(t) W(t) .
\end{aligned}
$$
that

According to Assumption 1 and Lemma 1, it can deduce

$$
\begin{aligned}
2 z^{T}(t) A_{1} F(t) & =\left(A_{1}^{T} z((t))\right)^{T} F(t)+F^{T}(t)\left(A_{1}^{T} z(t)\right) \\
& \leq z^{T}(t) A_{1} A_{1}^{T} z(t)+F^{T}(t) F(t) \\
& \leq z^{T}(t) A_{1} A_{1}^{T} z(t)+l_{1}^{2} z^{T}(t) z(t) .
\end{aligned}
$$

Analogously, we have that 


$$
\begin{aligned}
2 z(t)^{T} B_{1} G(t-\tau) & =\left(B_{1}^{T} z(t)\right)^{T} G(t-\tau)+G^{T}(t-\tau)\left(B_{1}^{T} z(t)\right) \\
& \leq z^{T}(t) B_{1} B_{1}^{T} z(t)+G^{T}(t-\tau) G(t-\tau) \\
& \leq z^{T}(t) B_{1} B_{1}^{T} z(t)+l_{2}^{2}\|\tilde{x}(t)\|^{2}+\sum_{i=1}^{n} l_{2 i}^{2}\left|x_{i}(t-\tau)-\tilde{x}_{i}(t)\right|^{2} \\
& \leq z^{T}(t) B_{1} B_{1}^{T} z(t)+l_{2}^{2}\|\widetilde{x}(t)\|^{2}+\sum_{i=1}^{n} 2 l_{2 i}^{2}\left(\left|x_{i}(t-\tau)\right|^{2}+\left|\tilde{x}_{i}(t)\right|^{2}\right) \\
& \leq z^{T}(t) B_{1} B_{1}^{T} z(t)+3 l_{2}^{2}\|\widetilde{x}(t)\|^{2}+\sum_{i=1}^{n} 2 l_{2 i}^{2}\left|x_{i}(t-\tau)\right|^{2} \\
& \leq z^{T}(t) B_{1} B_{1}^{T} z(t)+3 l_{2}^{2}\|\widetilde{x}(t)\|^{2}+\sum_{i=1}^{n} 4 l_{2 i}^{2}\left(\left|e_{i}(t-\tau)\right|^{2}+\left|\tilde{x}_{i}(t-\tau)\right|^{2}\right) \\
& \leq z^{T}(t)\left(B_{1} B_{1}^{T}+3 l_{2}^{2} I\right) z(t)+4 l_{2}^{2} z^{T}(t-\tau) z(t-\tau), \\
2 z(t)^{T} W(t) & =z^{T}(t) W(t)+W^{T}(t) z(t) \\
& \leq \varepsilon_{1} z^{T}(t) z(t)+\varepsilon_{1}^{-1} W(t)^{T} W(t) .
\end{aligned}
$$

Applying Assumption 2 and combining with (35)-(38), we get that

$$
\begin{aligned}
\dot{V}(t) & \leq z^{T}(t)\left[C_{1}+C_{1}^{T}+A_{1} A_{1}^{T}+B_{1} B_{1}^{T}+\left(l_{1}^{2}+3 l_{2}^{2}+g_{1}\right) I\right] z(t)-\left(g_{1}-\varepsilon_{1}\right) z^{T}(t) z(t)+4 l_{2}^{2} z^{T}(t-\tau) z(t-\tau)+\varepsilon_{1}^{-1} W^{T}(t) W(t) \\
& \leq-\left(g_{1}-\varepsilon_{1}\right) V(z(t))+4 l_{2}^{2} V(z(t-\tau))+\varepsilon_{1}^{-1}\|\omega\|_{\left[t_{i}, t\right]}^{2} .
\end{aligned}
$$

Let $h(t)=\varepsilon_{1}^{-1}\|\omega\|_{\left[t_{i}, t\right]}^{2}$. From Lemma 2, for any holds. On account of the continuity of $V(z(t))$, one has $t_{i} \leq t<t_{i}+\vartheta_{i}$, the following inequality

$$
\begin{aligned}
V(z(t)) & \leq\left[V\left(z\left(t_{i}\right)\right)+\int_{t_{i}}^{t} e^{-\tilde{r}_{1}\left(s-t_{i}\right)} h(s) \mathrm{d} s\right] e^{\tilde{r}_{1}\left(t-t_{i}\right)} \\
& =\left[V\left(z\left(t_{i}\right)\right)+\int_{t_{i}}^{t} e^{r_{1}\left(s-t_{i}\right)} h(s) \mathrm{d} s\right] e^{-r_{1}\left(t-t_{i}\right)},
\end{aligned}
$$

$$
\begin{aligned}
V\left(z\left(t_{i}+\vartheta_{i}\right)\right) & \leq V\left(z\left(t_{i}\right)\right) e^{-r_{1} \vartheta_{i}}+e^{-r_{1} \vartheta_{i}} \int_{t_{i}}^{t_{i}+\vartheta_{i}} e^{r_{1}\left(s-t_{i}\right)} h(s) \mathrm{d} s \\
& \leq V\left(z\left(t_{i}\right)\right) e^{-r_{1} \vartheta_{i}}+\varepsilon_{1}^{-1} r_{1}^{-1}\|\omega\|_{\left[t_{i}, t_{i}\right]+\vartheta_{i}}^{2} .
\end{aligned}
$$

$$
\begin{aligned}
\dot{V}(z(t)) & =2 z^{T}(t) \dot{z}(t) \\
& =2 z(t)^{T} C_{2} z(t)+2 z(t)^{T} A_{1} F(t)+2 z(t)^{T} B_{1} G(t-\tau)+2 z(t)^{T} W(t) \\
& \leq z^{T}(t)\left[2 C_{2}+A_{1} A_{1}^{T}+B_{1} B_{1}^{T}+\left(l_{1}^{2}+3 l_{2}^{2}-g_{2}\right) I\right] z(t)+\left(g_{2}+\varepsilon_{2}\right) z^{T}(t) z(t)+4 l_{2}^{2} z^{T}(t-\tau) z(t-\tau)+\varepsilon_{2}^{-1} W^{T}(t) W(t) \\
& \leq\left(g_{2}+\varepsilon_{2}\right) V(z(t))+4 l_{2}^{2} V(z(t-\tau))+\varepsilon_{2}^{-1}\|\omega\|_{\left[t_{i}+\vartheta_{i}, t\right]}^{2} .
\end{aligned}
$$

Let $\tilde{h}(t)=\varepsilon_{2}^{-1}\|\omega\|_{\left[t_{i}+\vartheta_{i}, t\right]}^{2}$. By Lemma 2, for any $t_{i}+\vartheta_{i} \leq t<t_{i+1}$, we get

$$
V(z(t)) \leq\left[V\left(z\left(t_{i}+\vartheta_{i}\right)\right)+\int_{t_{i}+\vartheta_{i}}^{t} e^{-r_{2}\left(s-t_{i}-\vartheta_{i}\right)} \widetilde{h}(s) \mathrm{d} s\right] \times e^{r_{2}\left(t-t_{i}-\vartheta_{i}\right)} .
$$


Since $V(z(t))$ is continuous, substituting (41) into (43), one has

$$
\begin{aligned}
V\left(z\left(t_{i+1}\right)\right) & \leq V\left(z\left(t_{i}+\vartheta_{i}\right)\right) e^{r_{2}\left(t_{i+1}-t_{i}-\vartheta_{i}\right)}+e^{\left(t_{i+1}-t_{i}-\vartheta_{i}\right)} \int_{t_{i}+\vartheta_{i}}^{t_{i+1}} e^{-r_{2}\left(s-t_{i}-\vartheta_{i}\right)} \tilde{h}(s) \mathrm{d} s \\
& \leq V\left(z\left(t_{i}+\vartheta_{i}\right)\right) e^{r_{2}\left(t_{i+1}-t_{i}-\vartheta_{i}\right)}+\varepsilon_{2}^{-1} r_{2}^{-1} e^{r_{2}\left(t_{i+1}-t_{i}-\vartheta_{i}\right)}\|\omega\|_{\left[t_{i}+\vartheta_{i}, t_{i+1}\right]}^{2} \\
& \leq V\left(z\left(t_{i}\right)\right) e^{-\left(r_{1}+r_{2}\right) \vartheta_{i}+r_{2}\left(t_{i+1}-t_{i}\right)}+\left(\varepsilon_{1}^{-1} r_{1}^{-1}+\varepsilon_{2}^{-1} r_{2}^{-1}\right) e^{r_{1} \vartheta_{i}-\left(r_{1}+r_{2}\right) \vartheta_{i}+r_{2}\left(t_{i+1}-t_{i}\right)}\|\omega\|_{\left[t_{i}, t_{i+1}\right]}^{2} .
\end{aligned}
$$

Let $\eta=\varepsilon_{1}^{-1} r_{1}^{-1}+\varepsilon_{2}^{-1} r_{2}^{-1}, \eta_{1}=\varepsilon_{1}^{-1} r_{1}^{-1}$, and $\rho_{i j}(t) \triangleq-\left(r_{1}+\right.$ $\left.r_{2}\right) \sum_{k=j}^{i-1} \vartheta_{k}+r_{2}\left(t-t_{j}\right)$.
By using (44), we get

$$
\begin{aligned}
V\left(z\left(t_{i}\right)\right) \leq & V\left(z\left(t_{i-1}\right)\right) e^{-\left(r_{1}+r_{2}\right) \vartheta_{i-1}+r_{2}\left(t_{i}-t_{i-1}\right)}+\eta e^{r_{1} \vartheta_{i-1}-\left(r_{1}+r_{2}\right) \vartheta_{i-1}+r_{2}\left(t_{i}-t_{i-1}\right)}\|\omega\|_{\left[t_{i-1}, t_{i}\right]}^{2} \\
\leq & V\left(z\left(t_{i-2}\right)\right) e^{-\left(r_{1}+r_{2}\right)\left(\vartheta_{i-2}+\vartheta_{i-1}\right)+r_{2}\left(t_{i}-t_{i-2}\right)}+\eta\left[e^{r_{1} \vartheta_{i-2}-\left(r_{1}+r_{2}\right)\left(\vartheta_{i-2}+\vartheta_{i-1}\right)+r_{2}\left(t_{i}-t_{i-2}\right)}+e^{\left.r_{1} \vartheta_{i-1}-\left(r_{1}+r_{2}\right) \vartheta_{i-1}+r_{2}\left(t_{i}-t_{i-1}\right)\right]}\|\omega\|_{\left[t_{i-2}, t_{i}\right]}^{2}\right. \\
\leq & V\left(z\left(t_{i-3}\right)\right) e^{-\left(r_{1}+r_{2}\right)\left(\vartheta_{i-3}+\vartheta_{i-2}+\vartheta_{i-1}\right)+r_{2}\left(t_{i}-t_{i-3}\right)}+\eta\left[e^{r_{1} \vartheta_{i-3}-\left(r_{1}+r_{2}\right)\left(\vartheta_{i-3}+\vartheta_{i-2}+\vartheta_{i-1}\right)+r_{2}\left(t_{i}-t_{i-3}\right)}+e^{r_{1} \vartheta_{i-2}-\left(r_{1}+r_{2}\right)\left(\vartheta_{i-2}+\vartheta_{i-1}\right)+r_{2}\left(t_{i}-t_{i-2}\right)}\right. \\
& \left.+e^{r_{1} \vartheta_{i-1}-\left(r_{1}+r_{2}\right) \vartheta_{i-1}+r_{2}\left(t_{i}-t_{i-1}\right)}\right]\|\omega\|_{\left[t_{i-3}, t_{i}\right]}^{2} \\
& \ldots \\
\leq & V\left(z\left(t_{0}\right)\right) e^{\rho_{i, 0}\left(t_{i}\right)}+\eta \sum_{j=0}^{i-1} e^{r_{1} \vartheta_{j}+\rho_{i, j}\left(t_{i}\right)}\|\omega\|_{\left[t_{0}, t_{i}\right]}^{2} .
\end{aligned}
$$

Similar to the reasoning process of (44) and combined with (43) and (45), for any $t_{i}+\vartheta_{i} \leq t<t_{i+1}$, the following inequality

$$
V(z(t)) \leq V\left(z\left(t_{0}\right)\right) e^{\rho_{i+1,0}(t)}+\eta \sum_{j=0}^{i} e^{r_{1} \vartheta_{j}+\rho_{i+1, j}(t)}\|\omega\|_{\left[t_{0}, t\right]}^{2},
$$

holds.

Combining (40) and (45), for any $t_{i} \leq t<t_{i}+\vartheta_{i}$, we get

$$
\begin{aligned}
V(z(t)) & \leq V\left(z\left(t_{i}\right)\right) e^{-r_{1}\left(t-t_{i}\right)}+\varepsilon_{1}^{-1} r_{1}^{-1}\|\omega\|_{\left[t_{i}, t\right]}^{2} \\
& \leq V\left(z\left(t_{i}\right)\right)+\varepsilon_{1}^{-1} r_{1}^{-1}\|\omega\|_{\left[t_{i}, t\right]}^{2} \\
& \leq V\left(z\left(t_{0}\right)\right) e^{\rho_{i, 0}(t)}+\left(\eta_{1}+\eta \sum_{j=0}^{i-1} e^{r_{1} \vartheta_{j}+\rho_{i, j}(t)}\right)\|\omega\|_{\left[t_{0}, t\right]}^{2} .
\end{aligned}
$$

From (46) and (47), one can see that the stabilization relies largely on $\rho_{i j}(t)$. Hence, it is utterly momentous to evaluate $\rho_{i j}(t)$.

By (33), there exists $\lambda \in\left(0, r_{1}\right)$, such that

$$
\gamma_{\text {inf }}=\inf _{i \in \mathbb{N}}\left\{\frac{\vartheta_{i}}{\Delta_{i}}\right\}=\frac{\lambda+r_{2}}{r_{1}+r_{2}}
$$

thus,

$$
\frac{\lambda+r_{2}}{r_{1}+r_{2}} \Delta_{i} \leq \vartheta_{i}, \quad i \in \mathbb{N} .
$$

By using (49) and the expression of $\rho_{i, j}(t)$, for any $t_{i} \leq t<t_{i}+\vartheta_{i}$ and $i>j$, we get

$$
\begin{aligned}
\rho_{i j}(t) & \leq-\left(\lambda+r_{2}\right) \sum_{k=j}^{i-1} \Delta_{k}+r_{2}\left(t-t_{j}\right) \\
& =-\left(\lambda+r_{2}\right)\left(t_{i}-t_{j}\right)+r_{2}\left(t-t_{j}\right) \\
& \leq-\lambda\left(t-t_{j}\right)+\left(\lambda+r_{2}\right) \vartheta_{i} .
\end{aligned}
$$

For any $t_{i}+\vartheta_{i} \leq t<t_{i+1}, i \geq j$. Based on the definition of $\rho_{i j}(t)$ and the derivation of (50), we have

$$
\rho_{i+1, j}(t) \leq \rho_{i+1, j}\left(t_{i+1}\right) \leq-\lambda\left(t_{i+1}-t_{j}\right) .
$$

Combining (47) and (50), for any $t_{i} \leq t<t_{i}+\vartheta_{i}$, we obtain that 


$$
\begin{aligned}
V(z(t)) & \leq V\left(z\left(t_{0}\right)\right) e^{-\lambda\left(t-t_{0}\right)+\left(\lambda+r_{2}\right) \vartheta_{i}}+\left[\eta_{1}+\eta \sum_{j=0}^{i-1} e^{r_{1} \vartheta_{j}+\left(r_{2}+\lambda\right) \vartheta_{i}-\lambda\left(t-t_{j}\right)}\right] \times\|\omega\|_{\left[t_{0}, t\right]}^{2} \\
& \leq V\left(z\left(t_{0}\right)\right) e^{-\lambda\left(t-t_{0}\right)+\left(\lambda+r_{2}\right) \vartheta_{\max }}+\left[\eta_{1}+\eta \sum_{j=1}^{i} e^{\left(r_{1}+r_{2}+\lambda\right) \vartheta_{\max }-j \lambda \vartheta_{\min }}\right] \times\|\omega\|_{\left[t_{0}, t\right]}^{2} \\
& \leq V\left(z\left(t_{0}\right)\right) e^{-\lambda\left(t-t_{0}\right)+\left(\lambda+r_{2}\right) \vartheta_{\max }}+\eta\left[1+\frac{e^{\left(r_{1}+r_{2}+\lambda\right) \vartheta_{\max }}}{1-e^{-\lambda \vartheta_{\min }}}\right]\|\omega\|_{\left[t_{0}, t\right]}^{2} .
\end{aligned}
$$

Similarly, combining (46) and (51), for any $t_{i}+\vartheta_{i} \leq t<t_{i+1}$, one has

$$
\begin{aligned}
V(z(t)) & \leq V\left(z\left(t_{0}\right)\right) e^{-\lambda\left(t_{i+1}-t_{0}\right)}+\eta \sum_{j=0}^{i} e^{r_{1} \vartheta_{j}-\lambda\left(t_{i+1}-t_{j}\right)}\|\omega\|_{\left[t_{0}, t\right]}^{2} \\
& \leq V\left(z\left(t_{0}\right)\right) e^{-\lambda\left(t-t_{0}\right)}+\eta \sum_{j=1}^{i+1} e^{r_{1} \vartheta_{\max }-j \lambda \vartheta_{\min }}\|\omega\|_{\left[t_{0}, t\right]}^{2} \\
& \leq V\left(z\left(t_{0}\right)\right) e^{-\lambda\left(t-t_{0}\right)}+\eta \frac{e^{r_{1} \vartheta_{\max }}}{1-e^{-\lambda \vartheta_{\min }}}\|\omega\|_{\left[t_{0}, t\right]}^{2} .
\end{aligned}
$$

From (52) and (53), for any $t \geq t_{0}$, we have

$$
V(z(t)) \leq \xi_{1} V\left(z\left(t_{0}\right)\right) e^{-\lambda\left(t-t_{0}\right)}+\xi_{2}\|\omega\|_{\left[t_{0}, t\right]}^{2},
$$

where $\quad \xi_{1}=e^{\left(\lambda+r_{2}\right) 9_{\max }}$ and $\xi_{2}=\eta\left[1+\left(e^{\left(r_{1}+r_{2}+\lambda\right) \vartheta_{\max }} / 1\right.\right.$ $\left.\left.-e^{-\lambda \theta_{\min }}\right)\right]$.

Hence, for any $t \geq t_{0}$,

$$
\|z(t)\| \leq \sqrt{\xi_{1}}\left\|z\left(t_{0}\right)\right\| e^{-(\lambda / 2)\left(t-t_{0}\right)}+\sqrt{\xi_{2}}\|\omega\|_{\left[t_{0}, t\right]},
$$

which means that neural network (1) is the stabilization to exponential ISS under observer-based T-APIC (4).

In addition, from (49), one has

$$
\frac{\lambda+r_{2}}{r_{1}+r_{2}} \sum_{j=0}^{i} \Delta_{j} \leq \sum_{j=0}^{i} \vartheta_{j}, \quad i \in \mathbb{N} .
$$

Therefore, we get

$$
R_{u}^{*}=\frac{r_{2}}{r_{1}+r_{2}}<\frac{\lambda+r_{2}}{r_{1}+r_{2}} \leq R_{\min } .
$$

The deducing procedure is finished.

Remark 5. Assume $\left\{9_{i}\right\}$ satisfies (29), and for any $i \in N$, the following inequality,

$$
\frac{\lambda+r_{2}}{r_{1}+r_{2}} \Delta_{i} \leq \vartheta_{i}<\Delta_{i}
$$

holds, where $\lambda$ is a sufficiently small constant satisfying $0<\lambda<r_{1}$; then, we can deduce (33), and so, Theorem 1 is still true.

Because (9) is a switched system under observer-based APIC $\left\{K_{1}, K_{2},\left\{t_{i}\right\},\left\{\vartheta_{i}\right\}\right\}$, we will next give a sufficient condition for the exponential ISS of (9) by using the converse average dwell time.

Set notation $N[s, t)$ represents the control times of the observer-based APIC during $[s, t)$.

Theorem 2. Assume that $\left\{t_{i}\right\}$ is given to satisfy (28) and the converse average dwell time condition for appropriate $₫>0$, $d \geq 0, \tilde{i} \geq 1$, and for any $t_{i} \leq t<t_{i+1}, i \geq \widetilde{i}$,

$$
N[s, t) \geq \frac{t-s}{\varpi}-d, \quad t \geq s \geq t_{0} .
$$

Under Assumptions 1 and 2, (9) can realize stabilization to exponential ISS via observer-based T-APIC $\left\{K_{1}, K_{2}\right.$, $\left.\left\{t_{i}\right\},\left\{\vartheta_{i}\right\}\right\}$, if $\left\{\vartheta_{i}\right\}$ satisfies (29) and

$$
R_{u}^{*}=\frac{r_{2}}{r_{1}+r_{2}}<\frac{\vartheta_{\min }}{\varpi}<1,
$$

where $r_{1}$ and $r_{2}$ are the same as those in Theorem 1.

Moreover, we get

$$
R_{\min }>R_{u}^{*}
$$

Proof. Likewise, let $V(z(t))=z^{T}(t) z(t)$. The definition of function $\rho_{i j}(t)$ is same as that in Theorem 1 . Meanwhile, by Assumptions 1 and 2 and the reasoning process of Theorem 1 , we still get (40)-(47).

From (60), there is $\lambda$ satisfying $0<\lambda<r_{1}$ and the following equation:

$$
\frac{r_{1}+r_{2}}{\lambda+r_{2}} \vartheta_{\min }=\emptyset .
$$

Then, converse average dwell time condition (59) can be rewritten as, for any $t_{i} \leq t<t_{i+1}, i \geq \widetilde{i}$,

$$
N[s, t) \geq \frac{t-s}{\left(r_{1}+r_{2}\right) /\left(\lambda+r_{2}\right) \vartheta_{\text {min }}}-d, \quad t \geq s \geq t_{0} .
$$

Let $d_{1}=\vartheta_{\min }\left(r_{1}+r_{2}\right) d+\left(\lambda+r_{2}\right) \Delta_{\max }$, where $\Delta_{\max }=$ $\max \left\{\Delta_{i}\right\}=\max \left\{t_{i+1}-t_{i}\right\}, i \in \mathbb{N}$. When $i \geq \widetilde{i}$ and for any $t_{i} \leq t<t_{i+1}, \quad t_{j}+\vartheta_{j} \leq s<t_{j+1} \quad$ satisfying $i>j, \quad$ one has $N[s, t)=i-j$. Similar to Theorem 1 , we now need to estimate $\rho_{i j}(t)$.

For any $t_{i} \leq t<t_{i}+\vartheta_{i}$, by using (63), we have 


$$
\begin{aligned}
\rho_{i j}(t) & =-\left(r_{1}+r_{2}\right) \sum_{k=j}^{i-1} \vartheta_{k}+r_{2}\left(t-t_{j}\right) \\
& \leq-\left(r_{1}+r_{2}\right)(i-j) \vartheta_{\min }+r_{2}\left(t-t_{j}\right) \\
& \leq-(t-s)\left(\lambda+r_{2}\right)+\vartheta_{\min }\left(r_{1}+r_{2}\right) d+r_{2}\left(t-t_{j}\right) \\
& =-\left(t-t_{j}+t_{j}-s\right)\left(\lambda+r_{2}\right)+\vartheta_{\min }\left(r_{1}+r_{2}\right) d+r_{2}\left(t-t_{j}\right) \\
& \leq-\lambda\left(t-t_{j}\right)+\left(\lambda+r_{2}\right) \Delta_{j}+\vartheta_{\min }\left(r_{1}+r_{2}\right) d \\
& \leq-\lambda\left(t-t_{j}\right)+d_{1} .
\end{aligned}
$$

For any $t_{i}+\vartheta_{i} \leq t<t_{i+1}$, from the definition of $\rho_{i, j}(t)$ and the reasoning process of (64), we get

$$
\rho_{i+1, j}(t) \leq \rho_{i+1, j}\left(t_{i+1}\right) \leq-\lambda\left(t_{i+1}-t_{j}\right)+d_{1} .
$$

When $i \geq \widetilde{i}$, for any $t_{i} \leq t<t_{i}+\vartheta_{i}$, based on (47), (64), and the derivation of (47), we deduce

$$
V(z(t)) \leq e^{d_{1}} V\left(z\left(t_{0}\right)\right) e^{-\lambda\left(t-t_{0}\right)}+\eta\left(1+\frac{e^{r_{1} 9_{\max }+d_{1}}}{1-e^{-\lambda 9_{\min }}}\right)\|\omega\|_{\left[t_{0}, t\right]}^{2} .
$$

Analogously, combining (46) and (65), we get

$$
V(z(t)) \leq e^{d_{1}} V\left(z\left(t_{0}\right)\right) e^{-\lambda\left(t-t_{0}\right)}+\eta \frac{e^{r_{1} 9_{\max }+d_{1}}}{1-e^{-\lambda \vartheta_{\min }}}\|\omega\|_{\left[t_{0}, t\right]}^{2},
$$

for any $t_{i}+\vartheta_{i} \leq t<t_{i+1}$.

Hence, $\forall t \geq t \underset{i}{-}$; from (66) and (67), one has

$V(z(t)) \leq e^{d_{1}} V\left(z\left(t_{0}\right)\right) e^{-\lambda\left(t-t_{0}\right)}+\eta\left(1+\frac{e^{r_{1} 9_{\max }+d_{1}}}{1-e^{-\lambda 9_{\min }}}\right)\|\omega\|_{\left[t_{0}, t\right]}^{2}$.

Then, we analyse the case of $t_{0} \leq t<t \widetilde{i}$.

According to (40), (46), (47), and the definition of $\rho_{i j}(t)$, it follows that

(1) When $t_{0} \leq t<t_{0}+\vartheta_{0}$,

$$
\begin{aligned}
V(z(t)) & \leq V\left(z\left(t_{0}\right)\right) e^{-r_{1}\left(t-t_{0}\right)}+e^{-r_{1}\left(t-t_{0}\right)} \int_{t_{0}}^{t} e^{r_{1}\left(s-t_{0}\right)} h(s) \mathrm{d} s \\
& \leq V\left(z\left(t_{0}\right)\right) e^{-r_{1}\left(t-t_{0}\right)}+\eta_{1}\|\omega\|_{\left[t_{0}, t\right]}^{2} \\
& \leq V\left(z\left(t_{0}\right)\right) e^{-\lambda\left(t-t_{0}\right)}+\eta_{1}\|\omega\|_{\left[t_{0}, t\right]}^{2} .
\end{aligned}
$$

(2) When $t_{0}+\vartheta_{0} \leq t<t_{1}$,

$$
\begin{aligned}
V(z(t)) & \leq V\left(z\left(t_{0}\right)\right) e^{\rho_{1,0}(t)}+\eta e^{r_{1} \vartheta_{0}+\rho_{1,0}(t)}\|\omega\|_{\left[t_{0}, t\right]}^{2} \\
& \leq V\left(z\left(t_{0}\right)\right) e^{-\left(r_{1}+r_{2}\right) \vartheta_{\min }+r_{2}\left(t-t_{0}\right)}+\eta\left(1+e^{r_{1} \vartheta_{\max }+r_{2}\left(t_{1}-t_{0}\right)-\left(r_{1}+r_{2}\right) \vartheta_{\min }}\right)\|\omega\|_{\left[t_{0}, t\right]}^{2} .
\end{aligned}
$$

(3) When $t_{1} \leq t<t_{1}+\vartheta_{1}$,

$$
\begin{aligned}
V(z(t)) & \leq V\left(z\left(t_{0}\right)\right) e^{\rho_{1,0}(t)}+\left(\eta_{1}+\eta e^{r_{1} \vartheta_{0}+\rho_{1,0}(t)}\right)\|\omega\|_{\left[t_{0}, t\right]}^{2} \\
& \leq V\left(z\left(t_{0}\right)\right) e^{-\left(r_{1}+r_{2}\right) \vartheta_{\min }+r_{2}\left(t-t_{0}\right)}+\eta\left(1+e^{r_{1} \vartheta_{\max }+r_{2}\left(t_{1}+\vartheta_{1}-t_{0}\right)-\left(r_{1}+r_{2}\right) \vartheta_{\min }}\right)\|\omega\|_{\left[t_{0}, t\right]}^{2} .
\end{aligned}
$$

(4) When $t_{1}+\vartheta_{1} \leq t<t_{2}$,

$$
\begin{aligned}
V(z(t)) & \leq V\left(z\left(t_{0}\right)\right) e^{\rho_{2,0}(t)}+\eta \sum_{j=0}^{1} e^{r_{1} \vartheta_{j}+\rho_{2, j}(t)}\|\omega\|_{\left[t_{0}, t\right]}^{2} \\
& \leq V\left(z\left(t_{0}\right)\right) e^{-\left(r_{1}+r_{2}\right) 29_{\min }+r_{2}\left(t-t_{0}\right)}+\eta\left(1+e^{r_{1} 9_{\max }+r_{2}\left(t_{2}-t_{0}\right)} \sum_{j=1}^{2} e^{-\left(r_{1}+r_{2}\right) j \vartheta_{\min }}\right)\|\omega\|_{\left[t_{0}, t\right]}^{2} .
\end{aligned}
$$

(5) When $t_{2} \leq t<t_{2}+\vartheta_{2}$, 


$$
\begin{aligned}
V(z(t)) & \leq V\left(z\left(t_{0}\right)\right) e^{\rho_{2,0}(t)}+\left(\eta_{1}+\eta \sum_{j=0}^{1} e^{r_{1} \vartheta_{j}+\rho_{2, j}(t)}\right)\|\omega\|_{\left[t_{0}, t\right]}^{2} \\
& \leq V\left(z\left(t_{0}\right)\right) e^{-\left(r_{1}+r_{2}\right) 2 \vartheta_{\min }+r_{2}\left(t-t_{0}\right)}+\eta\left(1+e^{r_{1} 9_{\max }+r_{2}\left(t_{2}+\vartheta_{2}-t_{0}\right)} \sum_{j=1}^{2} e^{-\left(r_{1}+r_{2}\right) j \vartheta_{\min }}\right)\|\omega\|_{\left[t_{0}, t\right]}^{2} .
\end{aligned}
$$

By inducing, we get

(6) When $t_{i-1} \leq t<t_{i-1}+\vartheta_{i-1}$,

$$
\begin{aligned}
& V(z(t)) \leq V\left(z\left(t_{0}\right)\right) e^{\beta_{i-1,0}(t)}+\left(\eta_{1}+\eta \sum_{j=0}^{\tilde{i}-2} e^{r_{1} \vartheta_{j}+\beta_{i-1, j}}(t)\right) \times\|\omega\|_{\left[t_{0}, t\right]}^{2} \\
& \leq V\left(z\left(t_{0}\right)\right) e^{-\left(r_{1}+r_{2}\right) \tilde{(i-1)} \vartheta_{\min }+r_{2}\left(t-t_{0}\right)}+\eta\left(1+e^{r_{1} \vartheta_{\max }+r_{2}\left(\underset{i-1}{+\vartheta_{i-1}}-t_{0}\right)} \sum_{j=1}^{\tilde{i}-1} e^{-\left(r_{1}+r_{2}\right) j \vartheta_{\min }}\right) \times\|\omega\|_{\left[t_{0}, t\right]}^{2} .
\end{aligned}
$$

(7) When $t_{i-1}+\vartheta_{i-1} \leq t<t_{i}$,

$$
\begin{aligned}
& V(z(t)) \leq V\left(z\left(t_{0}\right)\right) e^{\rho_{i, 0}(t)}+\eta \sum_{j=0}^{\tilde{i}-1} e^{r_{1} \vartheta_{j}+\beta_{i, j}(t)}\|\omega\|_{\left[t_{0}, t\right]}^{2} \\
& \leq V\left(z\left(t_{0}\right)\right) e^{-\left(r_{1}+r_{2}\right) \tilde{i} \vartheta_{\min }+r_{2}\left(t-t_{0}\right)}+\eta\left(1+e^{r_{1} \vartheta_{\max }+r_{2}\left(\hbar-t_{0}\right)} \sum_{j=1}^{\tilde{i}} e^{-\left(r_{1}+r_{2}\right) j \vartheta_{\min }}\right)\|\omega\|_{\left[t_{0}, t\right]}^{2} .
\end{aligned}
$$

Let $\xi_{3}=\max \left\{1, e^{-\left(r_{1}+r_{2}\right) \vartheta_{\min }+\left(r_{2}+\lambda\right)\left(t_{i}-t_{0}\right)}\right\}$. From the above derivation, for any $t \in\left[t_{0}, t_{i}\right)$, we have

$$
V(z(t)) \leq \xi_{3} V\left(z\left(t_{0}\right)\right) e^{-\lambda\left(t-t_{0}\right)}+\eta\left(1+\frac{e^{r_{1} \vartheta_{\max }+r_{2}\left(t-t_{0}\right)-\left(r_{1}+r_{2}\right) \vartheta_{\min }}}{1-e^{-\left(r_{1}+r_{2}\right) \vartheta_{\min }}}\right)\|\omega\|_{\left[t_{0}, t\right]}^{2}
$$

Let $\alpha=\eta\left(1+\left(e^{r_{1} \vartheta_{\max }+r_{2}\left(t-t_{0}\right)-\left(r_{1}+r_{2}\right) \vartheta_{\min }} / 1-e^{-\left(r_{1}+r_{2}\right) \vartheta_{\min }}\right)\right)$ and $\beta=\eta\left(1+\left(e^{r_{1} \vartheta_{\max }+d_{1}} / 1-e^{-\lambda v_{\min }}\right)\right)$. Hence, for any $t \geq t_{0}$, one has

$$
V(z(t)) \leq \xi_{1}^{*} V\left(z\left(t_{0}\right)\right) e^{-\lambda\left(t-t_{0}\right)}+\xi_{2}^{*}\|\omega\|_{\left[t_{0}, t\right]}^{2},
$$

where $\xi_{1}^{*}=\max \left\{e^{d_{1}}, \xi_{3}\right\}$ and $\xi_{2}^{*}=\max \{\alpha, \beta\}$.

So, for any $t \geq t_{0}$,

$$
\|z(t)\| \leq \sqrt{\xi_{1}^{*}}\left\|z\left(t_{0}\right)\right\| e^{-(\lambda / 2)\left(t-t_{0}\right)}+\sqrt{\xi_{2}^{*}}\|\omega\|_{\left[t_{0}, t\right]},
$$

which indicates that (1) achieves stabilization to exponential ISS under observer-based T-APIC (4).

Next, we estimate $R_{\min }$.

From (59), it follows that

$$
\omega(i+1) \geq \sum_{j=0}^{i} \Delta_{j}-d \omega
$$

which means that

$$
\varpi \geq \limsup _{i \longrightarrow \infty} \frac{\sum_{j=0}^{i} \Delta_{j}-\mathrm{d} \omega}{i+1}=\limsup _{i \longrightarrow \infty} \frac{\sum_{j=0}^{i} \Delta_{j}}{i+1} .
$$

It follows from (30), (60), and (80) that

$$
R_{\min } \geq \min _{\left\{t_{i}\right\},\left\{\vartheta_{i}\right\}}\left\{\frac{\vartheta_{\text {min }}}{\lim _{i \longrightarrow \infty} \sup \left(\sum_{j=0}^{i} \Delta_{j} /(i+1)\right)}\right\} \geq \frac{\vartheta_{\min }}{\omega}>R_{u}^{*} .
$$

The deducing procedure is finished. 
Remark 6. When $\left\{t_{i}\right\}$ is given to satisfy (28) or satisfy (28) and converse average dwell time (59), by Theorem 1 and Theorem 2, there exists nontrival $\left\{\vartheta_{i}\right\}$, such that (1) is stabilized to exponential ISS via observer-based T-APIC (4). Also, we get that MATR $R_{\min }>R_{u}^{*}$. Comparing (59) with (60), it is worth mentioning that if converse average dwell time (59) holds, we can use the average dwell time $₫$ of $\left\{\Delta_{i}\right\}$ substitute for the single $\Delta_{i}$ in (58) to deduce observer-based T-APIC $\left(K_{1}, K_{2},\left\{t_{i}\right\},\left\{\vartheta_{i}\right\}\right)$.

\section{Case 2. $\left\{\vartheta_{i}\right\}$ is given to satisfy (29).}

Here, we will give homologous criteria to ensure that (1) is stabilized to exponential ISS via observer-based T-APIC (4) and estimate $R_{\min }$.

Theorem 3. Suppose $\left\{\vartheta_{i}\right\}$ is given to satisfy (29) and Assumptions 1 and 2 hold. (1) is stabilized to exponential ISS via observer-based T-APIC (4), if $\left\{t_{i}\right\}$ satisfies (28) and one of the following conditions:

(a)

$$
1<\gamma_{\text {inf }}^{-1}=\min _{i \in \mathbb{N}}\left\{\frac{\Delta_{i}}{\vartheta_{i}}\right\}<\left(R_{u}^{*}\right)^{-1}
$$

(b) For appropriate $\lambda$ satisfying $0<\lambda<r_{1}$ and any $i \in \mathbb{N}$,

$$
\vartheta_{i}<\Delta_{i} \leq \frac{r_{1}+r_{2}}{\lambda+r_{2}} \vartheta_{i}
$$

(c) Converse average dwell time (59) holds and $₫$ satisfies

$$
\vartheta_{\min }<\omega<\vartheta_{\min } \cdot\left(R_{u}^{*}\right)^{-1},
$$

$r_{1}$ and $r_{2}$ in the above conditions are the same as those in Theorem 1. Moreover, we can obtain that $R_{\min }>R_{u}^{*}$.

Proof. For (a) and (b), it can be similar to the proof in Theorem 1, and for (c), by using the similar proof of Theorem 2, we can get the result.

Remark 7. From Theorems $1-3$, it is not difficult to find that $R_{u}^{*}=r_{2} /\left(r_{1}+r_{2}\right)$ is an extremely significant index for $R_{\min }$ of observer-based T-APIC (4). In addition, one can see that neural network (1) realizes stabilization to exponential ISS via observer-based T-APIC (4) when $R_{u}^{*}<R_{\min }$.

Corollary 1. Under Assumptions 1 and 2, for any $i \in \mathbb{N}$, if $\vartheta_{i}=\vartheta$ and $\left\{t_{i}\right\}$ satisfy

$$
t_{i+1}<t_{i}+\left(1+\frac{r_{1}}{r_{2}}\right) \vartheta
$$

then system (1) realizes stabilization to exponential ISS via observer-based T-APIC (4).

Proof. From (85), we can deduce

$$
1>\min _{i \in \mathbb{N}}\left\{\frac{\vartheta}{\Delta_{i}}\right\}>\frac{r_{2}}{r_{1}+r_{2}} .
$$

Then, by (a) in Theorem 3, it follows that Corollary 2 is true.

PIC as an especial case of APIC, next, a corresponding result obtained by the observer-based PIC will be given.

Corollary 2. Under Assumptions 1 and 2, if control period T and control width $\vartheta$ of observer-based PIC satisfy

$$
\vartheta<T<\vartheta+\frac{r_{1}}{r_{2}} \vartheta
$$

then (1) can achieve stabilization to exponential ISS via the observer-based PIC.

Proof. Let $\vartheta_{i}=\vartheta$ and $T$ be the maximum value of $\Delta_{i}$ in (82), namely, $T=\max \left\{\Delta_{i}\right\}$. According to (82) of Theorem 3 and condition (87), Corollary 2 is correct.

\section{Stabilization to Exponential ISS via Observer- Based E-APIC}

Compared with the time-triggered control mechanism, the event-triggered control mechanism can not only save unnecessary adjustment times but also control intensity is more appropriate. Therefore, in Theorem 4, we propose the observer-based E-APIC to realize the exponential ISS of the system (1).

The following is the construction of the observer-based E-APIC.

Suppose Assumptions 1 and 2 hold. The observer-based E-APIC depends on two indices: threshold value $\delta_{\max }>1$ and check period $\Delta>0$, which is generally a comparatively great real number.

Let $V^{T}(z(t))=z^{T}(t) z(t)$. To ensure that the observerbased E-APIC satisfies (28) and (29), let $\left\{\vartheta_{i}\right\}$ satisfy

$$
0<\frac{\ln \delta_{\max }}{r_{1}}<\vartheta_{\min }=\min \left\{\vartheta_{i}\right\} \leq \vartheta_{i} \leq \Delta-\frac{\ln \delta_{\max }}{r_{2}} .
$$

The event-triggered mechanism is as follows:

$t_{i+1}= \begin{cases}\min \left\{t: t \in \widehat{\psi}_{i}\left(t_{i}+\vartheta_{i}, t_{i}+\Delta\right]\right\}, & \text { if } \widehat{\psi}_{i}\left(t_{i}+\vartheta_{i}, t_{i}+\Delta\right] \neq \varnothing ; \\ t_{i}+\Delta, & \text { if } \widehat{\psi}_{i}\left(t_{i}+\vartheta_{i}, t_{i}+\Delta\right]=\varnothing,\end{cases}$

where

$$
\widehat{\psi}_{i}(s, t] \triangleq\left\{\theta: s<\theta \leq t, V(z(\theta)) \geq \delta_{\max } V(z(s))+\xi_{m}\|\omega\|_{[s, \theta]}^{2}\right\},
$$

with $\xi_{m} \geq \max \left\{\varepsilon_{1}^{-1} r_{1}^{-1}, \varepsilon_{2}^{-1} r_{2}^{-1}\right\}, 0<\varepsilon_{1}<g_{1}, 0<\varepsilon_{2}<g_{2}$, and $r_{1}$ and $r_{2}$ are the same as those in Theorem 1 .

Remark 8. Since $\omega \in L_{\infty, \text { loc }}$, replacing $\|\omega\|_{\left[t_{i}+\vartheta_{i}, t\right]}$ with $\|\omega\|_{\infty}$ will simplify the calculation in (88) and (89).

The event-triggered control is closely related to the system state, that is, the controller is only activated when the systematic deviation reaches or exceeds the preset value 
given. The systematic deviation is usually expressed as $\|e(t)\|=\left\|x(t)-x\left(t_{i}\right)\right\|$, and the trigger condition is $\|e(t)\| \geq m$, where $m$ stands for the threshold value satisfying $m>0$ and $t_{i}>t$. Moreover, $t_{i+1}=\min \left\{t: t>t_{i},\{\|e(t)\| \geq m\}\right\}$. Since the state of the system studied can not be obtained completely, the error system between the observer and the plant is constructed, and the corresponding augmented system is constructed by using the observed value and the error. Significantly, there is a special case that $\{\|e(t)\|<m\}$ always holds, which indicates that the trigger condition has not appeared, that is, the controller has not been activated, which may lead to system instability. The check period proposed in [30] can effectively keep from this situation. Introducing a comparatively great check period to the observer-based E-APIC means eventhough the trigger condition is not satisfied during a check period, there will be a control input at the end of the check period to ensure the ISS of the system.

Theorem 4. Under Assumptions 1 and 2, (1) can realize stabilization to exponential ISS via observer-based E-APIC (88) and (89), and the MATR $R_{\min }$ of the observer-based E-APIC satisfies

$$
\frac{\vartheta_{\min }}{\Delta} \leq R_{\min } \leq \frac{\widetilde{r}_{2} r_{1} \vartheta_{\min }}{\left(\widetilde{r}_{2}+r_{1}\right) \ln \delta_{\max }},
$$

where $\tilde{r}_{2}$ is a positive constant satisfying $\tilde{r}_{2}>r_{2}$ and $-\widetilde{r}_{2}+\left(g_{2}+\varepsilon_{2}^{-1}+\varepsilon_{2}^{-1} \xi_{m}^{-1}\right)+4 l_{2}^{2} \leq 0$.

Proof. From (88) and (89), one can see that the interval between two adjacent controls $\Delta_{i}=t_{i+1}-t_{i}>\vartheta_{i}>$ $\left(\ln \delta_{\max } / r_{1}\right)>0$, which means the observer-based E-APIC will not be triggered countless times in a limited time. So, there is no Zeno behavior under the observer-based E-APIC. Furthermore, one can see that $\left\{\vartheta_{i}\right\}$ is nontrival.

For any $t_{i}+\vartheta_{i} \leq t<t_{i+1}$, from (42), we have

$\dot{V}(z(t)) \leq\left(g_{2}+\varepsilon_{2}\right) V(z(t))+4 l_{2}^{2} V(z(t-\tau))+\varepsilon_{2}^{-1}\|\omega\|_{\left[t_{i}+\vartheta_{i}, t\right]}^{2}$.

From (92), whenever $V(z(t)) \geq \xi_{m}\|\omega\|_{\left[t_{i}+9_{i}, t\right]}^{2}$, we get

$$
\dot{V}(z(t)) \leq\left(g_{2}+\varepsilon_{2}+\varepsilon_{2}^{-1} \xi_{m}^{-1}\right) V(z(t))+4 l_{2}^{2} V(z(t-\tau)) .
$$

Additionaly, since $r_{2} \geq g_{2}+\varepsilon_{2}+4 l_{2}^{2}$, apparently there exists $\widetilde{r}_{2}>0$, satisfying $\widetilde{r}_{2}>g_{2}+\varepsilon_{2}+\varepsilon_{2}^{-1} \xi_{m}^{-1}+4 l_{2}^{2}$; then, we can choose a positive constant $\widetilde{r}_{2}$ satisfying $\widetilde{r}_{2}>r_{2}$ and $-\widetilde{r}_{2}+g_{2}+\varepsilon_{2}+\varepsilon_{2}^{-1} \xi_{m}^{-1}+4 l_{2}^{2}<0$. has

By using Lemma 2 and (93), for any $t_{i}+\vartheta_{i} \leq t<t_{i+1}$, one

$$
V(z(t)) \leq V\left(z\left(t_{i}+\vartheta_{i}\right)\right) e^{\widetilde{r}_{2}\left(t-t_{i}-\vartheta_{i}\right)} .
$$

By the continuity of $V(z(t))$, we get

$$
\begin{aligned}
V\left(z\left(t_{i+1}\right)\right) & \leq e^{\tilde{r}_{2}\left(t_{i+1}-t_{i}-\vartheta_{i}\right)} V\left(z\left(t_{i}+\vartheta_{i}\right)\right) \\
& \leq e^{\tilde{r}_{2}\left(t_{i+1}-t_{i}-\vartheta_{i}\right)} V\left(z\left(t_{i}+\vartheta_{i}\right)\right)+\xi_{m}\|\omega\|_{\left[t_{i}+\vartheta_{i} t_{i+1}\right]}^{2} .
\end{aligned}
$$

On the one hand, by (89), if $\widehat{\psi}_{i}\left(t_{i}+\vartheta_{i}, t_{i}+\Delta\right] \neq \varnothing$, which means $t_{i+1} \leq t_{i}+\Delta$, one has

$$
V\left(z\left(t_{i+1}\right)\right)=\delta_{\max } V\left(z\left(t_{i}+\vartheta_{i}\right)\right)+\xi_{m}\|\omega\|_{\left[t_{i}+\vartheta_{i}, t_{i+1}\right]}^{2},
$$

since $V(z(t))$ is continuous.

Then, combining (95) and (96), we have

$$
\delta_{\max } \leq e^{\tilde{r}_{2}\left(t_{i+1}-t_{i}-9_{i}\right)},
$$

which implies that

$$
0<\frac{\ln \delta_{\max }}{\widetilde{r}_{2}} \leq t_{i+1}-t_{i}-\vartheta_{i} \leq \Delta-\vartheta_{\min },
$$

for any $i \in \mathbb{N}$.

On the other hand, if $\widehat{\psi}_{i}\left(t_{i}+\vartheta_{i}, t_{i}+\Delta\right]=\varnothing$, then $t_{i+1}=t_{i}+\Delta$. By (88), we get

$$
0<\frac{\ln \delta_{\max }}{\widetilde{r}_{2}}<\frac{\ln \delta_{\max }}{r_{2}} \leq \Delta-\vartheta_{i}=t_{i+1}-t_{i}-\vartheta_{i} \leq \Delta-\vartheta_{\min },
$$

for any $i \in \mathbb{N}$.

Hence, for any $i \in \mathbb{N}$, we get (98) holds. Combining (88) and (98), we can obtain

$$
0<\left(\frac{1}{r_{1}}+\frac{1}{\widetilde{r}_{2}}\right) \ln \delta_{\max }<t_{i+1}-t_{i} \leq \Delta .
$$

Next, we show that (1) is stabilized to exponential ISS by the observer-based E-APIC.

For any $t_{i} \leq t<t_{i}+\vartheta_{i}$, by (40), one has

$$
V(z(t)) \leq \delta_{\max } V\left(z\left(t_{i}\right)\right)+\eta_{1}\|\omega\|_{\left[t_{i}, t\right]}^{2} .
$$

In addition, from (41), we have

$$
V\left(z\left(t_{i}+\vartheta_{i}\right)\right) \leq V\left(z\left(t_{i}\right)\right) e^{-r_{1} \vartheta_{i}}+\eta_{1}\|\omega\|_{\left[t_{i}, t_{i}+\vartheta_{i}\right]}^{2} .
$$

Let $\xi=\max \left\{\eta_{1}, \xi_{m}\right\}$ and $\tilde{\xi}=\delta_{\max } \eta_{1}+\xi$. For any $t_{i}+\vartheta_{i} \leq t<t_{i+1}, i \in \mathbb{N}$, by (89) and (102), we get

$$
\begin{aligned}
V(z(t)) & \leq \delta_{\max } V\left(z\left(t_{i}+\vartheta_{i}\right)\right)+\xi_{m}\|\omega\|_{\left[t_{i}+\vartheta_{i}, t\right]}^{2} \\
& \leq \delta_{\max } e^{-r_{1} \vartheta_{i}} V\left(z\left(t_{i}\right)\right)+\tilde{\xi}\|\omega\|_{\left[t_{i}, t\right]}^{2} .
\end{aligned}
$$

Let $b_{i}=V\left(z\left(t_{i}\right)\right)$ and $p_{i}=\delta_{\max } e^{-r_{1} \vartheta_{i}}$, for any $i \in \mathbb{N}$; by (89) and the continuity of $V(z(t))$, one has

$$
b_{i+1} \leq p_{i} b_{i}+\tilde{\xi}\|\omega\|_{\left[t_{i}, t\right]}^{2} .
$$

Let $\tilde{\lambda}=r_{1} \vartheta_{\min }-\ln \delta_{\max }$ and $\widetilde{\xi}^{*}=\tilde{\xi}\left(1-e^{-\tilde{\lambda}}\right)^{-1}$. It follows from (88) that $\lambda>0$; in addition, by (104), for any $i \in \mathbb{N}$, we can obtain 


$$
\begin{aligned}
b_{i} & \leq e^{\sum_{j=0}^{i-1} \ln \delta_{\max }-r_{1} \vartheta_{j}} b_{0}+\tilde{\xi}\left(1+\sum_{j=1}^{i-1} e^{\sum_{k=j}^{i-1}\left(\ln \delta_{\max }-r_{1} \vartheta_{i}\right)}\right)\|\omega\|_{\left[t_{0}, t_{i}\right]}^{2} \\
& \leq e^{-\widetilde{\lambda i}} b_{0}+\tilde{\xi}\left(1+\sum_{j=1}^{i-1} e^{j\left(\ln \delta_{\max }-r_{1} \vartheta_{\min }\right)}\right)\|\omega\|_{\left[t_{0}, t_{i}\right]}^{2} \\
& \leq e^{-\tilde{\lambda i}_{i}} b_{0}+\widetilde{\xi}^{*}\|\omega\|_{\left[t_{0}, t_{i}\right]}^{2} .
\end{aligned}
$$

By using (101) and (103), for any $t_{i} \leq t \leq t_{i+1}, i \in \mathbb{N}$, we get

$$
V(z(t)) \leq \delta_{\max } V\left(z\left(t_{i}\right)\right)+\tilde{\xi}\|\omega\|_{\left[t_{i}, t\right]}^{2} .
$$

Combining (105) and (106), for any $t \geq t_{0}$, we get

$$
\begin{aligned}
V(z(t)) & \leq \delta_{\max } e^{-\tilde{\lambda} i} V\left(z\left(t_{0}\right)\right)+\left(\tilde{\xi}+\delta_{\max } \tilde{\xi}^{*}\right)\|\omega\|_{\left[t_{0}, t\right]}^{2} \\
& \leq \delta_{\max } \tilde{\lambda}^{\tilde{\lambda}} e^{(-\tilde{\lambda} / \Delta)\left(t-t_{0}\right)} V\left(z\left(t_{0}\right)\right)+\left(\widetilde{\xi}+\delta_{\max } \tilde{\xi}^{*}\right)\|\omega\|_{\left[t_{0}, t\right]}^{2} .
\end{aligned}
$$

Hence, for any $t \geq t_{0}$, by (107), we have

$$
\|z(t)\| \leq \sqrt{\delta_{\max } \tilde{e}^{\tilde{\lambda}}} e^{(-\tilde{\lambda} / 2 \Delta)}\left\|z\left(t_{0}\right)\right\|+\sqrt{\tilde{\xi}+\delta_{\max } \tilde{\xi}^{*}}\|\omega\|_{\left[t_{0}, t\right]} \text {, }
$$

which means that (1) realizes stabilization to exponential ISS under observer-based E-APIC (88) and (89). In addition, let $\vartheta_{i}=\vartheta_{\min }$ and combining (30) and (100), (91) holds. The deducing procedure is finished.

The following is the contrast among observer-based PIC, observer-based T-APIC, and observer-based E-APIC. For the convenience of exposition, denote by $R_{\min }^{P}, R_{\min }^{T}$, and $R_{\min }^{E}$, respectively, MATR of observer-based PIC, observerbased T-APIC, and observer-based E-APIC.

Set symbol $N_{P}(t), N_{T}(t)$, and $N_{E}(t)$, respectively, the control times of the observer-based PIC, the observer-based T-APIC, and the observer-based E-APIC on interval $\left[t_{0}, t\right)$. Let $\vartheta_{\min }^{T}$ and $\vartheta_{\min }^{E}$ denote the minimum activation time of observer-based T-APIC and observer-based E-APIC, respectively.

Theorem 5. Let $\vartheta_{i}=\vartheta, i \in \mathbb{N}$, in observer-based T-APIC and observer-based E-APIC as in observer-based PIC. Then, if Assumptions 1 and 2 hold, there is an observer-based E-APIC (88) and (89) satisfying

$$
R_{\min }^{E} \leq R_{u}^{*} \leq \min \left\{R_{\min }^{P}, R_{\min }^{T}\right\} .
$$

Proof. Follow Theorems 1-3 and Corollary 2; there exists a fully small $\varepsilon$ satisfying $0<\varepsilon \ll r_{1}$, such that $\min \left\{R_{\min }^{P}\right.$, $\left.R_{\min }^{T}\right\} \geq\left(r_{2}+\varepsilon\right) /\left(r_{1}+r_{2}\right)$. Let $\delta_{\max }=e^{r_{1} \widetilde{r}_{2} 9\left(r_{1}+r_{2}\right)-\left(\varepsilon+r_{2}\right)\left(\widetilde{r}_{2}+r_{1}\right)}$ in observer-based E-APIC (88) and (89); then, by (91) in Theorem 4, we have $R_{\min }^{E} \leq\left(\varepsilon+r_{2}\right) /\left(r_{1}+r_{2}\right)=R_{u}^{*}+$ $\varepsilon /\left(r_{1}+r_{2}\right)$. Hence, (109) holds when $\varepsilon \longrightarrow 0$.

\section{Theorem 6.}

(a) If $\sigma_{E} \triangleq\left(\left(1 / r_{1}\right)+\left(1 / r_{2}\right)\right) \ln \delta_{\text {max }} \geq T$, then, for any $t \geq t_{0}$, one has

$$
\left[\frac{t-t_{0}}{\Delta}\right] \leq N_{E}(t) \leq\left[\frac{t-t_{0}}{\sigma_{E}}\right] \leq N_{P}(t)
$$

(b) Let observer-based T-APIC satisfy $9_{\min }^{T}>0$ and converse average dwell time $N_{T}(t) \geq\left(t-t_{0}\right) / \sigma_{T}$, where $\sigma_{T} \triangleq\left(\left(r_{1}+r_{2}\right) /\left(\lambda+\widetilde{r}_{2}\right)\right) \vartheta_{\min }^{T}$ with $\lambda$ defined by (44). If $\ln \delta_{\max } \geq \sigma_{T}\left(\left(1 / r_{1}\right)+\left(1 / \widetilde{r}_{2}\right)\right)^{-1}$, then $N_{E}(t) \leq N_{T}(t)$.

(c) If the threshold value $\delta_{\max }$ in observer-based E-APIC (88) and (89) satisfies $\ln \delta_{\max } \geq \max \left\{\sigma_{T}, T\right\}$ $\left(\left(1 / r_{1}\right)+\left(1 / \widetilde{r}_{2}\right)\right)^{-1}$, then, for any $t \geq t_{0}$, we get

$$
N_{E}(t) \leq \min \left\{N_{P}(t), N_{E}(t)\right\} .
$$

Proof. By (100), we can derive (a). From (a) and $\sigma_{E}>\sigma_{T}$, we have (b), and (c) follows from (a) and (b).

Remark 9. By Theorem 6, the control times of observerbased E-APIC are less than those of observer-based PIC and observer-based T-APIC when we choose an appropriate $\delta_{\max }$.

\section{Example}

In this section, we provide a numerical example to simulate the validity of the obtained results.

Example 1. Consider a two-dimensional neural network with time delay and external disturbances, which is described as

$$
\left(\begin{array}{l}
x_{1}(t) \\
x_{2}(t)
\end{array}\right)=-\left(\begin{array}{ll}
1 & 0 \\
0 & 1
\end{array}\right)\left(\begin{array}{l}
x_{1}(t) \\
x_{2}(t)
\end{array}\right)+\left(\begin{array}{cc}
1 & 0.1 \\
0.1 & 1
\end{array}\right)\left(\begin{array}{l}
f_{1}\left(x_{1}(t)\right) \\
f_{2}\left(x_{2}(t)\right)
\end{array}\right)+\left(\begin{array}{cc}
-1 & 0.1 \\
0.1 & -1
\end{array}\right)\left(\begin{array}{l}
g_{1}\left(x_{1}(t-\tau)\right) \\
g_{2}\left(x_{2}(t-\tau)\right)
\end{array}\right)+\left(\begin{array}{l}
u_{11}(t) \\
u_{12}(t)
\end{array}\right)+\left(\begin{array}{l}
\omega_{1}(t) \\
\omega_{2}(t)
\end{array}\right) .
$$

Then, the corresponding state observer is designed by 


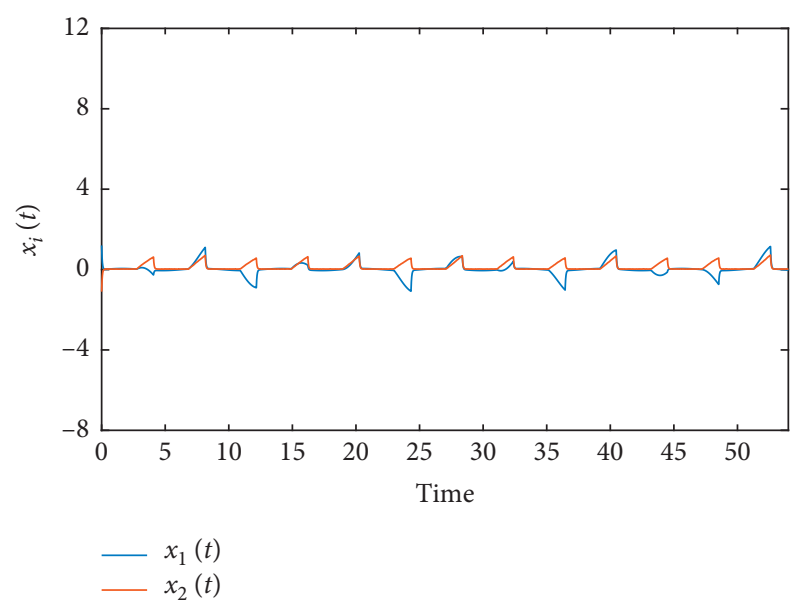

Figure 1: State trajectories $x_{i}(i=1,2)$ of system (112) under the observer-based T-APIC.

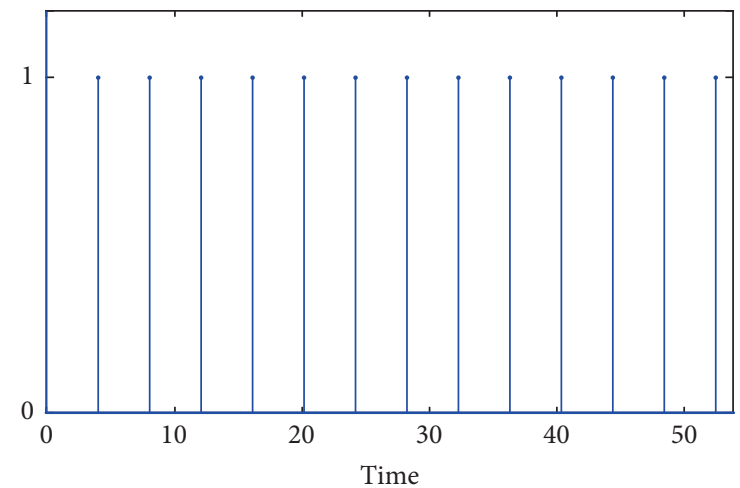

$\longrightarrow t_{i}$

FIGURE 2: The starting instant of each control of the observer-based T-APIC.

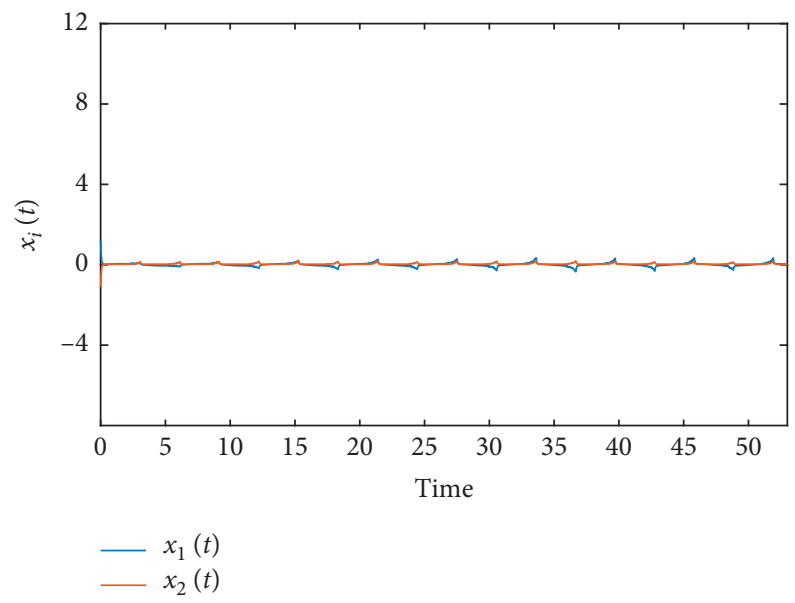

FIgURE 3: State trajectories $x_{i}(i=1,2)$ of system (112) under the observer-based T-APIC.

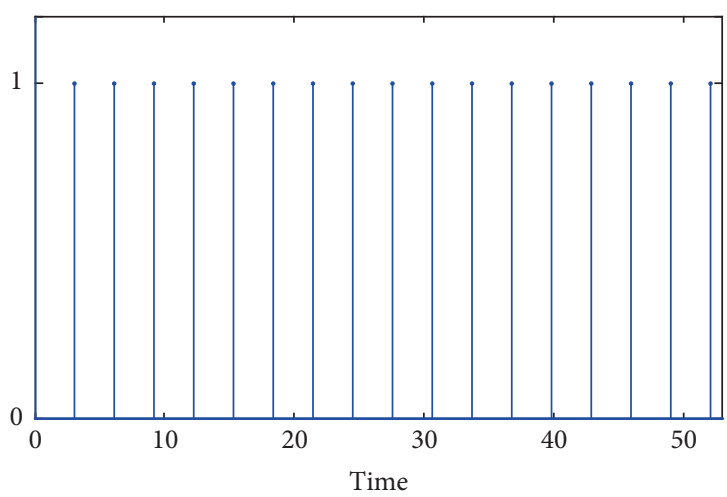

$\longrightarrow t_{i}$

FIGURE 4: The starting instant of each control of the observer-based T-APIC.

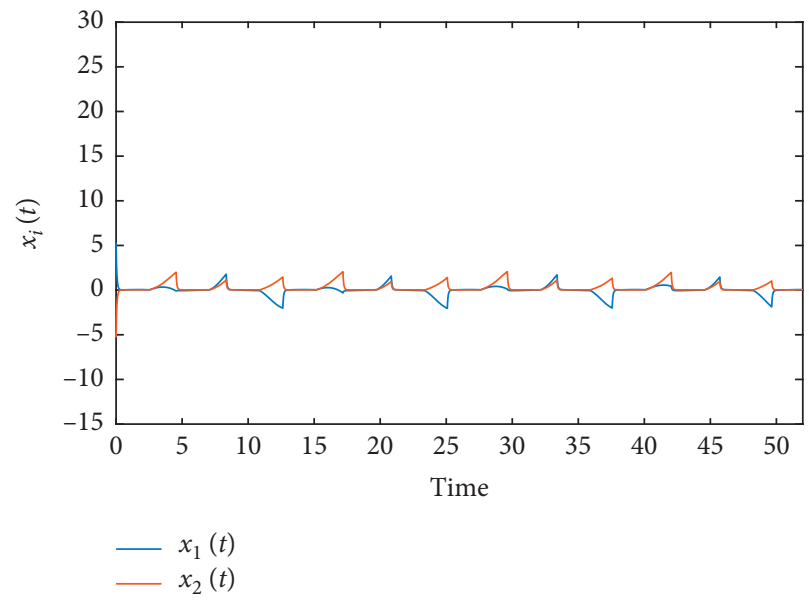

FIGURE 5: State trajectories $x_{i}(i=1,2)$ of system (112) under the observer-based E-APIC.

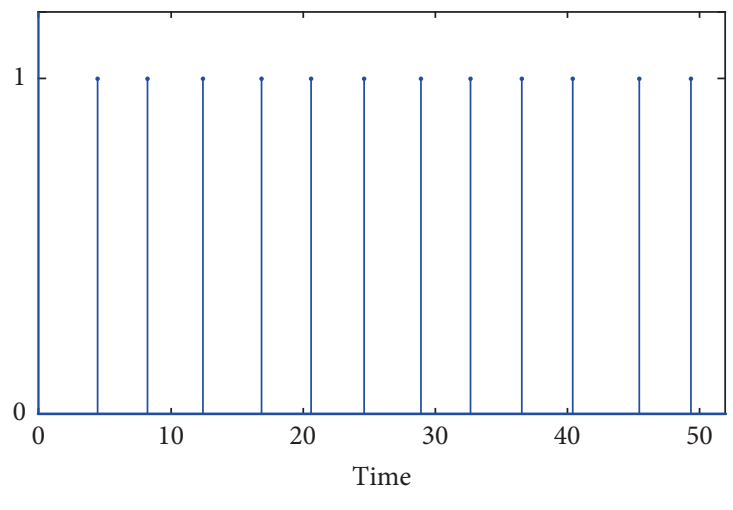

$t_{i}$

FIGURE 6: The starting instant of each control of the observer-based E-APIC. 


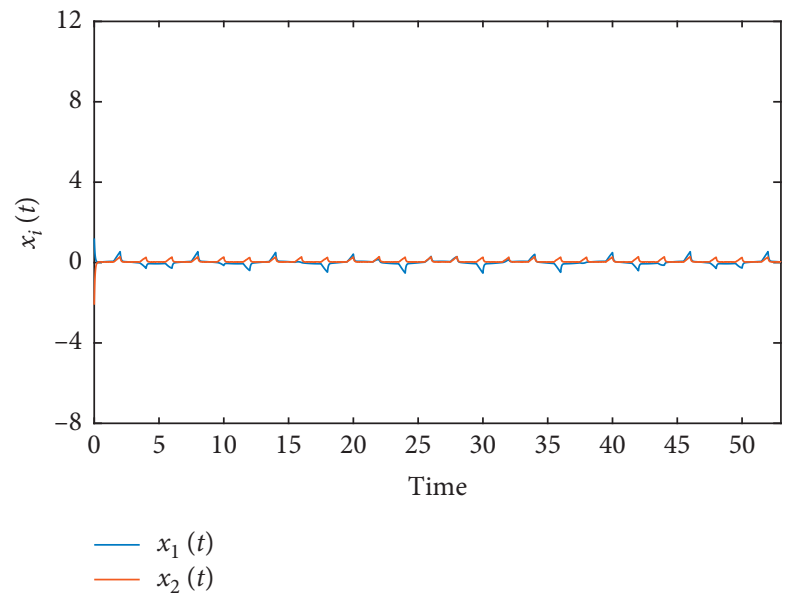

Figure 7: State trajectories $x_{i}(i=1,2)$ of system (112) under the observer-based PIC.

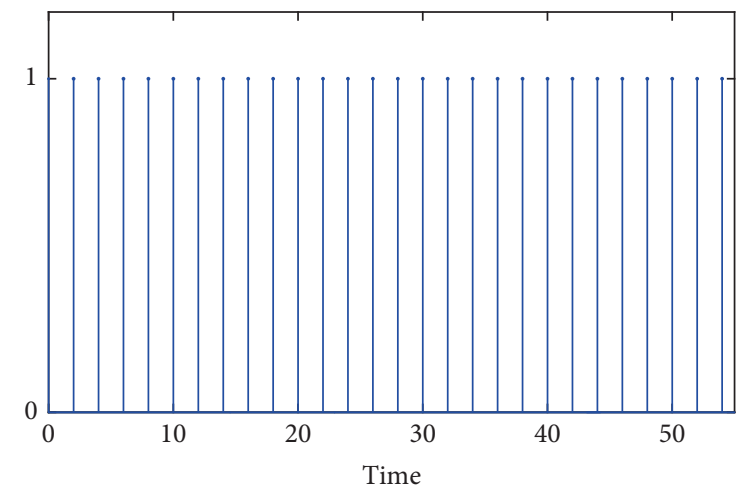

$\longrightarrow t_{i}$

FIGURE 8: The starting instant of each control of the observer-based PIC.

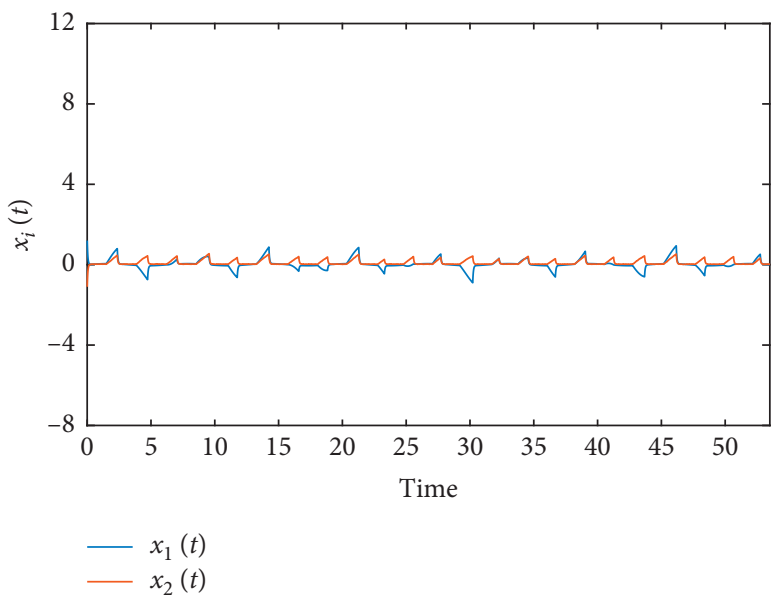

Figure 9: State trajectories $x_{i}(i=1,2)$ of system (112) under the observer-based T-APIC.

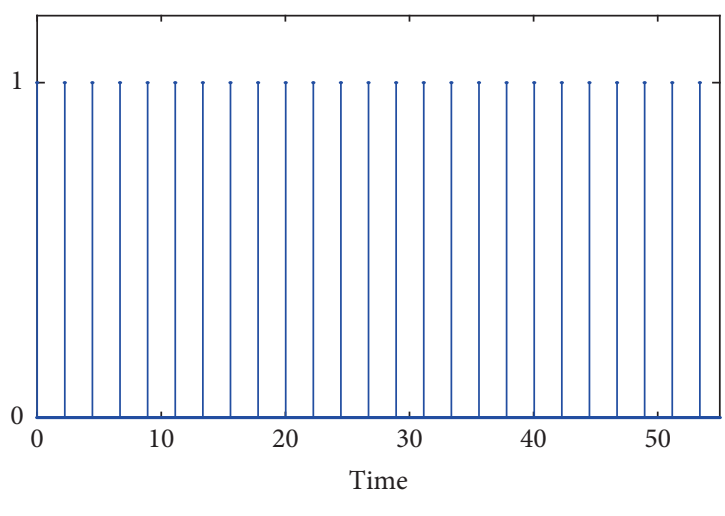

$\longrightarrow t_{i}$

FIgURE 10: The starting instant of each control of the observerbased T-APIC.

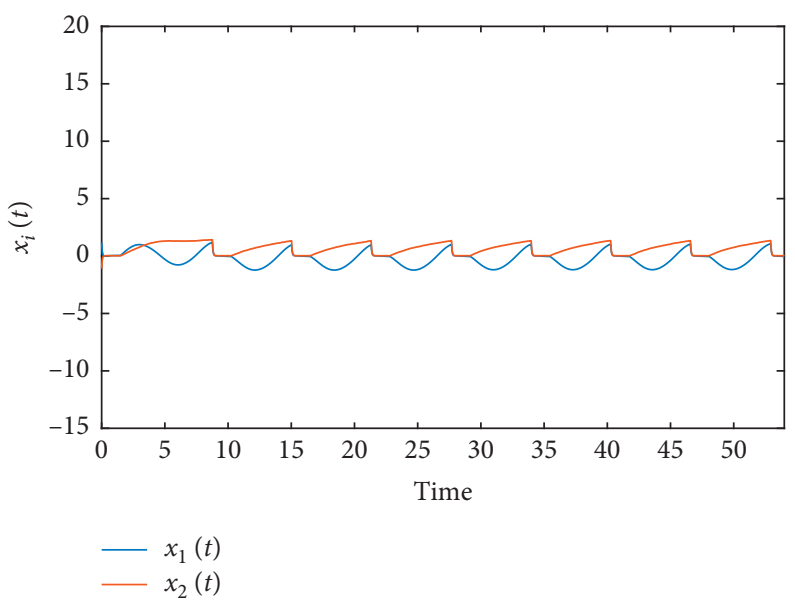

FIGURE 11: State trajectories $x_{i}(i=1,2)$ of system (112) under the observer-based E-APIC.

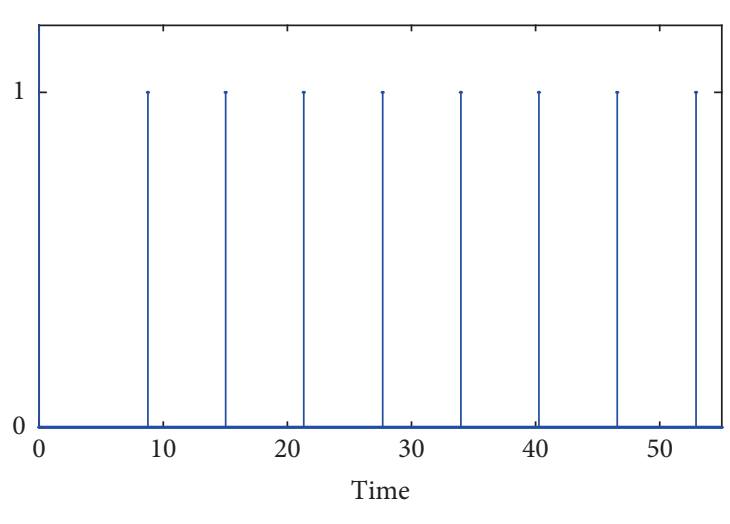

Figure 12: The starting instant of each control of the observerbased E-APIC. 
TABle 1: Contrasts among observer-based PIC, observer-based T-APIC, observer-based E-APIC.

\begin{tabular}{lccc}
\hline & Observer-based PIC & Observer-based T-APIC & Observer-based E-APIC \\
\hline$N(100)$ & 50 & 44 & 15 \\
$R(100)$ & $75 \%$ & $66 \%$ & $22 \%$ \\
& & $R_{u}^{*} \approx 58 \%$ & \\
\hline
\end{tabular}

$$
\left(\begin{array}{l}
\tilde{x}_{1}(t) \\
\tilde{x}_{2}(t)
\end{array}\right)=-\left(\begin{array}{ll}
1 & 0 \\
0 & 1
\end{array}\right)\left(\begin{array}{l}
\tilde{x}_{1}(t) \\
\tilde{x}_{2}(t)
\end{array}\right)+\left(\begin{array}{cc}
1 & 0.1 \\
0.1 & 1
\end{array}\right)\left(\begin{array}{l}
f_{1}\left(\tilde{x}_{1}(t)\right) \\
f_{2}\left(\tilde{x}_{2}(t)\right)
\end{array}\right)+\left(\begin{array}{cc}
-1 & 0.1 \\
0.1 & -1
\end{array}\right)\left(\begin{array}{l}
g_{1}\left(\tilde{x}_{1}(t)\right) \\
g_{2}\left(\tilde{x}_{2}(t)\right)
\end{array}\right)+\left(\begin{array}{l}
u_{21}(t) \\
u_{22}(t)
\end{array}\right) .
$$

Let

$$
\begin{aligned}
& f_{i}\left(x_{i}\right)=\sin \left(x_{i}\right), \quad i=1,2, \\
& g_{i}\left(x_{i}\right)=0.1 \tanh \left(x_{i}\right), \quad i=1,2,
\end{aligned}
$$

and the delay of $\tau$ is set to be $0.1,\left(\omega_{1}(t), \omega_{2}(t)\right)^{T}=$ $(\sin (t) \text {, rand }(1))^{T}$. Moreover, solving matrix inequalities (13) and (14), we have $g_{1}=9.9796, g_{2}=5.0204, K_{1}=-19.5 I$, and $K_{2}=-19.6 I$. Let $\varepsilon_{1}=\varepsilon_{2}=1$. By solving inequalities (31) and (32), we have $\widetilde{r}_{1} \geq-8.8$ and $r_{2} \geq 6.0604$, choose $\widetilde{r}_{1}=-5.28, r_{2}=7.42$, and then $r_{1}=\left|\widetilde{r}_{1}\right|=5.28$.

Here, let $t_{i+1}=t_{i}+4+0.55 \times$ rand (1). In order to satisfy the conditions in Theorem 1 , we choose $\vartheta_{i}=2.7+$ $0.1 \times \operatorname{rand}(1)$; then, neural network (112) can realize stabilization to exponential ISS via the observer-based T-APIC. The numerical simulations are shown in Figures 1 and 2 .

According to (28) and (59), let $t_{i+1}=t_{i}+3+\operatorname{rand}(1)$. If $\vartheta_{i}$ is designed as $\vartheta_{i}=2+\operatorname{rand}(1)$, then the conditions (29) and (60) of Theorem 2 can be satisfied, which means that neural network (112) can realize stabilization to exponential ISS via the observer-based T-APIC. The state trajectories of (112) and the starting instant of each control of the observerbased T-APIC are shown in Figures 3 and 4 .

Let the threshold value $\delta_{\max }=15$, check period $\Delta=10$, and $\xi_{m}=0.5 \geq \max \{0.1894,0.1348\}$. According to Theorem 4 , we choose $\vartheta_{i}=2.5+0.1 \times$ rand $(1)$; then, under the eventtriggered mechanism (89), system (112) realizes stabilization to exponential ISS. The numerical simulations are shown in Figures 5 and 6 .

To better compare observer-based PIC, observer-based T-APIC, and observer-based E-APIC, let control duration $\vartheta_{i}=1.5$ and the remaining parameters remain unchanged.

(a) For observer-based PIC, according to Corollary 2, let $T=2$. Then, neural network (112) can realize stabilization to exponential ISS via observer-based PIC. The state trajectories of (112) and the starting instant of each control of the observer-based PIC are shown in Figures 7 and 8, respectively.

(b) For observer-based T-APIC, according to Theorem 3 , let $\left\{t_{i}\right\}$ be designed as $t_{i+1}=t_{i}+2+0.566 \times$ rand(1). Then, neural network (112) can realize stabilization to exponential ISS via observer-based T-APIC. The numerical simulations refer to Figures 9 and 10, respectively.

(c) For observer-based E-APIC, let the threshold value $\delta_{\max }=15$, check period $\Delta=10$, and $\xi_{m}=0.5$. From
Theorem 4, system (112) is stabilization to exponential ISS under the event-triggered mechanism (89). The state trajectories of (112) and the starting instant of each control of the observer-based E-APIC are shown in Figures 11 and 12, respectively.

As follows, the three kinds of controllers are compared from the control times and activation time rate in the same time. Here, we extract the relevant data of $100 \mathrm{~s}$ in the numerical simulations, as given in Table 1.

From Table 1, one can see that the control times of observer-based E-APIC are least and the activation time rate of the observer-based E-APIC is lowest, which show that the observer-based E-APIC is superior to the observer-based T-APIC and the observer-based PIC in control times and activation time rate; in the meanwhile, we can get the observer-based T-APIC has some better performances than the observer-based PIC.

\section{Conclusions}

This study focuses on the exponential input-to-state stability of a class of neural networks with time delay and external disturbances under the observer-based APIC. Considering that the state of the system and the delay information may not be accurately obtained in practical application, a state observer independent of historical information is structured. For realizing exponential input-to-state stability of the studied network, the observer-based T-APIC is designed by using the infimum of single activation time rate and the condition of converse average dwell time, respectively. An observer-based E-APIC is advanced to solve the problem that the results obtained under the observer-based T-APIC may be conservative. In addition, the MATR of the observerbased T-APIC and the observer-based E-APIC are estimated. The results show that the observer-based E-APIC is superior to the observer-based PIC and the observer-based T-APIC in terms of control times and activation time rate, and the observer-based T-APIC is also superior to the observer-based PIC. Compared with the disturbances considered, the random disturbance makes the system have more abundant and interesting trajectories. Therefore, it is our next work to study the stability of neural networks with random disturbance under aperiodic intermittent control.

\section{Data Availability}

No data were used to support this study. 


\section{Conflicts of Interest}

The authors declare that there are no conflicts of interest.

\section{Acknowledgments}

This work was supported by the Natural Science Foundation of China (62072164 and 11704109) and Ministry of Nature Resources of China (KF-2018-08-031).

\section{References}

[1] Z. Liu, A. Chen, J. Cao, and L. Huang, "Existence and global exponential stability of periodic solution for BAM neural networks with periodic coefficients and time-varying delays," IEEE Transactions on Circuits and Systems I: Fundamental Theory and Applications, vol. 50, no. 9, pp. 1162-1173, 2003.

[2] B. S. Chen and J. Wang, "Global exponential periodicity of a class of recurrent neural networks with oscillating parameters and time-varying delays," IEEE Transactions on Neural Networks, vol. 16, no. 6, pp. 1440-1448, 2005.

[3] M. Zheng, L. Li, H. Peng, J. Xiao, Y. Yang, and Y. Zhang, "Fixed-time synchronization of memristive fuzzy BAM cellular neural networks with time-varying delays based on feedback controllers," IEEE Access, vol. 6, pp. 12085-12102, 2018.

[4] Y. Hayakawa and K. Nakajima, "Design of the inverse function delayed neural network for solving combinatorial optimization problems," IEEE Transactions on Neural Networks, vol. 21, no. 2, pp. 224-237, 2010.

[5] G. X. Lou and H. Z. Shi, "Face image recognition based on convolutional neural network," China Communications, vol. 17, no. 2, pp. 117-124, 2020.

[6] T. Zeng and S. W. Ji, "Deep convolutional neural networks for multi-instance multi-task learning," in Proceedings of the 2015 IEEE International Conference on Data Mining, pp. 579-588, Atlantic City, NJ, USA, 2015.

[7] T. Gong, T. Lee, C. Stephenson et al., "A comparison of loss weighting strategies for multi task learning in deep neural networks," IEEE Access, vol. 7, pp. 141627-141632, 2019.

[8] J. Chen, B. Chen, Z. Zeng, and P. Jiang, "Event-based synchronization for multiple neural networks with time delay and switching disconnected topology," IEEE Transactions on Cybernetics, 2020, In press.

[9] J. P. Ramirez, E. Garcia, and J. Alvarez, "Master-slave synchronization via dynamic control," Communications in Nonlinear Science and Numerical Simulation, vol. 80, Article ID 104977, 2020.

[10] O. Faydasicok, "A new Lyapunov functional for stability analysis of neutral-type Hopfield neural networks with multiple delays," Neural Networks, vol. 129, pp. 288-297, 2020.

[11] L. Wan and Q. H. Zhou, "Stability analysis of neutral-type Cohen-Grossberg neural networks with multiple time-varying delays," IEEE Access, vol. 8, pp. 27618-27623, 2020.

[12] Y.X. Guo and C. Xu, "Global asymptotic stability for a class of neural networks with time-varying delays," in Proceedings of the 2014 IEEE Chinese Guidance, Navigation and Control Conference, pp. 72-76, Vancouver, Canada, August 2014.

[13] H. Akça, V. Covachev, and Z. Covacheva, "Global asymptotic stability of Cohen-Grossberg neural networks of neural type," Journal of Mathematical Sciences, vol. 205, pp. 719-732, 2015.
[14] J. Lian and K. Zhang, "Exponential stability for switched Cohen-Grossberg neural networks with average dwell time," Nonlinear Dynamics, vol. 63, pp. 331-343, 2011.

[15] Y. Sheng, Y. Shen, and M. F. Zhu, "Delay-Dependent global exponential stability for delayed recurrent neural networks," IEEE Transactions on Neural Networks and Learning Systems, vol. 28, no. 12, pp. 2974-2984, 2017.

[16] G. Zhang and Z. Zeng, "Exponential stability for a class of memristive neural networks with mixed time-varying delays," Applied Mathematics and Computation, vol. 321, pp. 544-554, 2018.

[17] L. Liu, Q. X. Zhu, and L. C. Feng, "Lagrange stability for delayed recurrent neural networks with Markovian switching based on stochastic vector Halandy inequalities," Neurocomputing, vol. 275, pp. 1614-1621, 2018.

[18] E. N. Sanchez and J. P. Perez, "Input-to-state stability (ISS) analysis for dynamic neural networks," IEEE Transactions on Circuits and Systems I: Fundamental Theory and Applications, vol. 46, no. 11, pp. 1395-1398, 1999.

[19] B. Li, Z. Wang, and Q.-L. Han, "Input-to-state stabilization of delayed differential systems with exogenous disturbances: the event-triggered case," IEEE Transactions on Systems, Man, and Cybernetics: Systems, vol. 49, no. 6, pp. 1099-1109, 2019.

[20] Q. Zhu, J. Cao, and R. Rakkiyappan, "Exponential input-tostate stability of stochastic Cohen-Grossberg neural networks with mixed delays," Nonlinear Dynamics, vol. 79, pp. 10851089, 2015.

[21] Y. Zhang, J. Sun, and G. Feng, "Impulsive control of discrete systems with time delay," IEEE Transactions on Automatic Control, vol. 54, no. 4, pp. 830-834, 2009.

[22] X. Liu and K. Zhang, "Stabilization of nonlinear time-delay systems: distributed-delay dependent impulsive control," Systems \& Control Letters, vol. 120, pp. 17-22, 2018.

[23] N. Liu, J. Fang, W. Deng, Z. J. Wu, and G. Q. Ding, "Synchronization for a class of fractional-order linear complex networks via impulsive control," International Journal of Control, Automation and Systems, vol. 16, pp. 2839-2844, 2018.

[24] X. D. Li and X. L. Fu, "Lag Synchronization of chaotic delayed neural networks via impulsive control," IMA Journal of Mathematical Control and Information, vol. 29, no. 1, pp. 133-145, 2012.

[25] X. D. Li and S. J. Song, "Stabilization of delay systems: delaydependent impulsive control," IEEE Transactions Automatic Control, vol. 62, no. 1, 2017.

[26] C. Li, G. Feng, and X. Liao, "Stabilization of nonlinear systems via periodically intermittent control," IEEE Transactions on Circuits and Systems II: Express Briefs, vol. 54, no. 11, pp. 1019-1023, 2007.

[27] X. Liu and T. Chen, "Synchronization of complex networks via aperiodically intermittent pinning control," IEEE Transactions on Automatic Control, vol. 60, no. 12, pp. 3316-3321, 2015.

[28] S. Cai, X. Li, P. Zhou, and J. Shen, "Aperiodic intermittent pinning control for exponential synchronization of memristive neural networks with time-varying delays," Neurocomputing, vol. 332, pp. 249-258, 2019.

[29] B. Guo, P. Shi, and C. Zhang, "Aperiodically intermittent control for synchronization of stochastic coupled networks with semi-Markovian jump and time delays," Nonlinear Analysis: Hybrid Systems, vol. 38, Article ID 100938, 2020.

[30] B. Liu, M. Yang, T. Liu, and D. J. Hill, "Stabilization to exponential input-to-state stability via aperiodic intermittent 
control," IEEE Transactions on Automatic Control, vol. 66, no. 6, pp. 2913-2918, 2021.

[31] X. Chen and F. Hao, "Oberver-based event-triggered control for certain and uncertain linear systems," IMA Journal of Mathematical Control and Information, vol. 30, no. 4, pp. 527-542, 2013.

[32] Y. Wang and X. Li, "Impulsive observer and impulsive control for time-delays systems," Journal of the Franklin Lnstitute, vol. 357, no. 13, pp. 8529-8542, 2020.

[33] X. D. Li, H. T. Zhu, and S. J. Song, "Input-to-state stability of nonlinear systems using observer-based event-triggered impulsive control," IEEE Transactions on Systems, Man, and Cybernetics: Systems, 2020, In press.

[34] L. Liu, X. W. Ding, W. N. Zhou, and X. L. Li, "Global mean square exponential stability and stabilization of uncertain switched delay systems with Lévy noise and flexible switching signals," Journal of the Franklin Institute, vol. 356, no. 18, pp. 11520-11545, 2019. 